\title{
Review of passive polarimetric dehazing methods
}

\author{
Wenfei Zhang $\odot,{ }^{\text {a,b,* Jian Liang, }}{ }^{\mathrm{c}}$ Guomei Wang, ${ }^{\mathrm{a}}$ Huanian Zhang, ${ }^{\mathrm{a}, \mathrm{b}}$ and \\ Shenggui Fu $\odot^{\text {a, } *}$ \\ ${ }^{\text {a}}$ Shandong University of Technology, School of Physics and Optoelectronic Engineering, \\ Zibo, China \\ ${ }^{\mathrm{b}}$ Shandong Normal University, Collaborative Innovation Center of Light Manipulations and \\ Applications, Jinan, China \\ 'Shaanxi Normal University, School of Physics and Information Technology, Xi'an, China
}

\begin{abstract}
The images acquired in haze conditions are significantly degraded due to the presence of atmospheric particles. These images have low contrast, poor visibility, and some information is lost as well. These characteristics severely hinder the further processing and the applications in which the images are being used. The polarimetric dehazing methods are effective for direct imaging and enhancing the imaging quality. In addition, polarimetric dehazing methods have the capability to cope with haze and other turbid mediums. Therefore, the polarimetric dehazing methods are extensively developed and used in different applications due to their superior performance. We present in detail the principles, the implementation techniques, and the advancements in the polarimetric dehazing methods. We believe that this work is the first in-depth review on the passive polarimetric dehazing methods. (c) 2021 Society of Photo-Optical Instrumentation Engineers (SPIE) [DOI: 10.1117/1.OE.60.3.030901]
\end{abstract}

Keywords: polarimetric imaging; imaging through turbid media; image enhancement.

Paper 20201430V received Dec. 14, 2020; accepted for publication Feb. 26, 2021; published online Mar. 13, 2021.

\section{Introduction}

Haze is a common atmospheric phenomenon, especially in industrial areas. The haze not only poses a threat to human health but also degrades the performance of many technological systems, such as optical monitor systems and remote sensing applications. Similarly, the images acquired in such conditions are seriously degraded and have low contrast, which leads to information loss, thus resulting in the poor performance of outdoor optical devices. ${ }^{1,2}$ Therefore, it is critical to develop such techniques and methods, which are able to enhance the outdoor imaging quality acquired for various applications. There are various methods proposed in recent literature for enhancing the quality of images acquired in hazy environment. In general, according to the number of input images, the image dehazing methods can be classified into single image dehazing methods and multiple images. The classification of the dehazing methods is shown in Fig. 1.

The single image dehazing methods only need single input hazy image. Among this kind of dehazing methods, two categories can further be divided considering whether a physical model is used or not. The first is the image enhancement methods without any model, ${ }^{3-19}$ and the other is image restoration methods based on a physical imaging degradation model in turbid media. ${ }^{20-33}$ The image enhancement methods aim at highlighting the targets of interest and improving the contrast regardless of the cause of the image's quality degradation, such as histogram equalization, ${ }^{3-6}$ retinex ${ }^{7-9}$ wavelet transform, ${ }^{10-12}$ homomorphic filtering, ${ }^{13}$ and so on. In addition, these techniques are based on simple algorithms and are highly efficient, thus making them suitable for many applications. However, the dehazing capacity of image enhancement methods is intrinsically limited, and these methods are only suitable for thin and homogeneous haze conditions. Also, the image restoration methods are based on specific physical imaging degradation models. These methods estimate the elements that play a role in degrading the image quality, including atmospheric light and airlight. The dehazed image can be obtained by inversely

*Address all correspondence to Wenfei Zhang, zhangwenfei@sdut.edu.cn; Shenggui Fu, fushenggui@sdut.edu.cn 


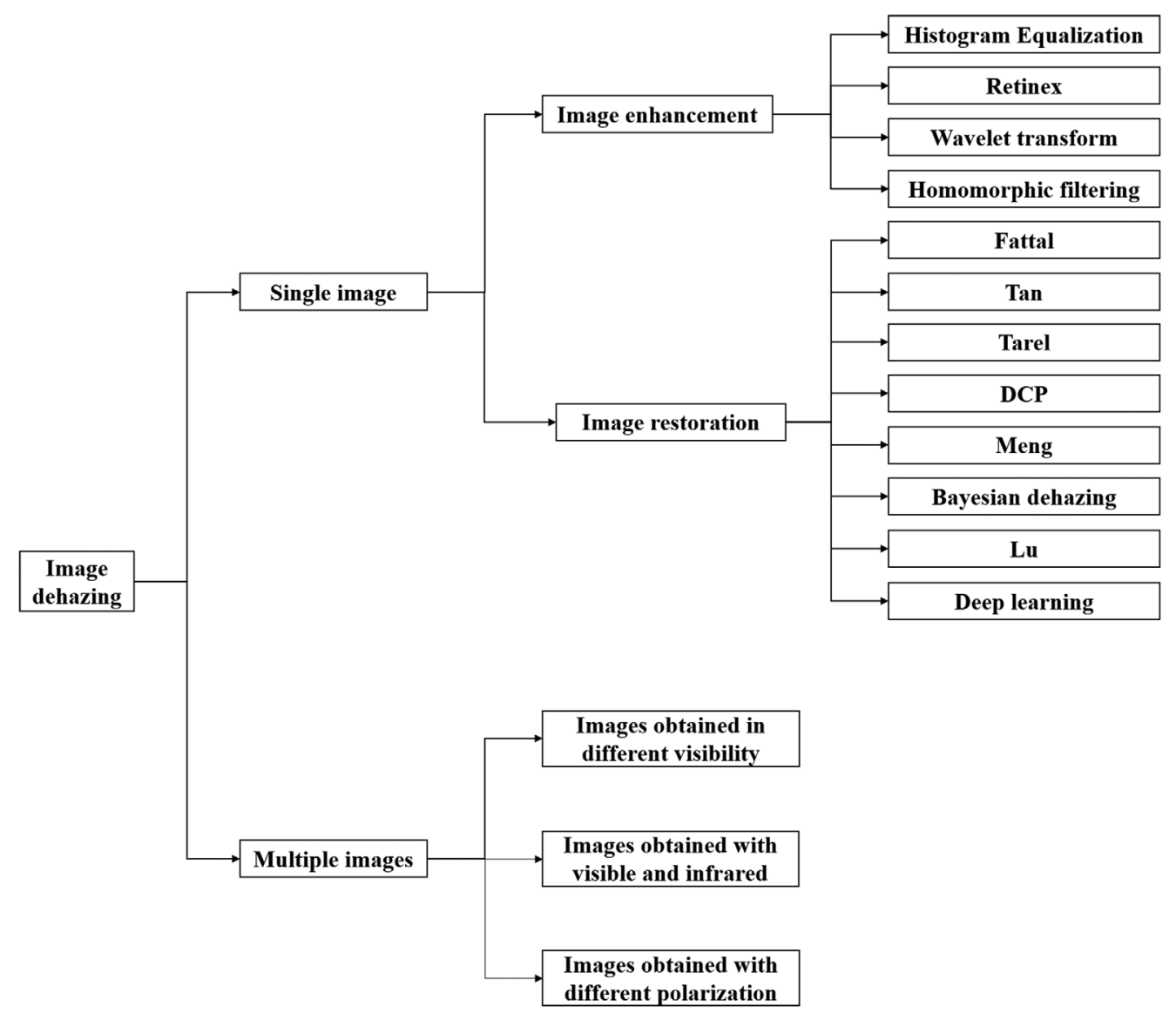

Fig. 1 The classification of image dehazing methods.

solving the physical model. The image restoration methods outperform the image enhancement methods in terms of dehazing capacity. ${ }^{34}$ However, the image restoration methods are difficult to derive due to the presence of many parameters, some assumptions or prior knowledge are required. It is notable that the image restoration methods are computationally complex as compared to image enhancement algorithms and are used in fewer applications, especially in real-time applications. Fattal, ${ }^{20}$ Tarel, ${ }^{21}$ Tan ${ }^{22}$ dark channel prior (DCP)-based, ${ }^{23-25}$ Meng, ${ }^{26}$ Bayesian dehazing, ${ }^{27,28} \mathrm{Lu}^{29}$ and deep learning-based ${ }^{32,33}$ are the most commonly discussed image restoration methods. The deep learning-based dehazing methods are intensely developed in last 5 years. Its main idea is to utilize convolutional neural network to learn and handle hazy image, finally estimate parameters based on traditional physical imaging degradation model to obtain dehazed image. The deep learning-based dehazing methods usually need plentiful clear and synthetized hazy image to train, which is a complex task.

The multiple images dehazing methods require more than one input image of the same scene. Until now, there are three kinds of multiple images dehazing methods: images obtained under different visibility, ${ }^{2,35-38}$ images obtained with visible and near-infrared camera, ${ }^{39,40}$ and images obtained with different polarizations. ${ }^{41-66}$ The depth discontinuities and the scene structure can be estimated through the changes of intensities of the images under different visibility with the same scene. Then, the image contrast can be enhanced via the estimated scene structure. This method is only suitable for static scenes. It is difficult to simultaneously acquire images with the same scenes under different visibility for dynamic scenes. The visible and near-infrared fusion dehazing methods rely on the fact that the near-infrared light propagates to a greater distance as compared to visible light, due to low scattering in turbid media. We can obtain an image of good quality by combining the color information of visible image and the high visibility of the nearinfrared image. However, the major obstacle in accomplishing this is the simultaneous acquisition of the visible image and the near-infrared image. Furthermore, the efficiency of the fusion 
algorithm is another challenge in combining these images. The polarization-based dehazing methods commonly known as polarimetric dehazing methods are designed on the basis of the fact that the airlight is partially polarized. So, the airlight radiance is estimated using multiple polarization images of the same scene for obtaining the dehazed image. The polarimetric dehazing methods show high information restoration capacity along with computational efficiency. With the assistance of polarimeter, the polarimetric dehazing methods have been widely developed for achieving good results. In this work, we present what we believe is the first in-depth review of the passive polarimetric dehazing methods. The major focus of this work is on the basic principles, implementation techniques, and the progress in the development of these methods. Thus, the subjective evaluation of dehazed image quality is not included in this work, which is another wide research topic and is out of scope of this paper.

\section{Imaging Model in Haze}

Imaging is a process in which the detector records the intensity and wavelength information of light emitted from a source or reflected from the target surfaces. In clear conditions, the emitted or reflected light from target surfaces is directly exposed on the detector without any scattering or attenuation. Consequently, the resultant image contains the detailed information of the target objects. However, in haze, due to the existence of haze particles, the imaging process is degraded due to the presence of particles that scatter the light. The well-known imaging degradation model used in the dehazing filed is shown in Fig. 2.1,2

According to the imaging degradation model, the image formation is described as

$$
I(x, y)=D(x, y)+A(x, y),
$$

where $I$ denotes the total radiance reaching the detector, and it is the sum of the direct transmission $D$ and the airlight $A$. $(x, y)$ denotes the pixel coordinates. The direct transmission $D$ is the attenuated object light $L$ :

$$
D(x, y)=L(x, y)+t(x, y),
$$

where $t$ represents the transmittance of the atmosphere associated with the distance between the scene and the camera, i.e., the amount of object light that reaches the detector. Note that the relationship between the transmittance and the wavelength is not considered. The object light $L$ is the desired result in dehazing. The airlight is the scattered atmospheric light:

$$
A(x, y)=A_{\infty}[1-t(x, y)],
$$

where $A_{\infty}$ represents the airlight radiance corresponding to an object at an infinite distance. Note that it is a global constant.

Due to the scattering and attenuation, the light from distant target objects is unable to reach the detector, resulting in poor visibility. Meanwhile, the airlight blends into the direct transmission and dominates the target signal, leading to low contrast. Therefore, the object light $L$, i.e.,

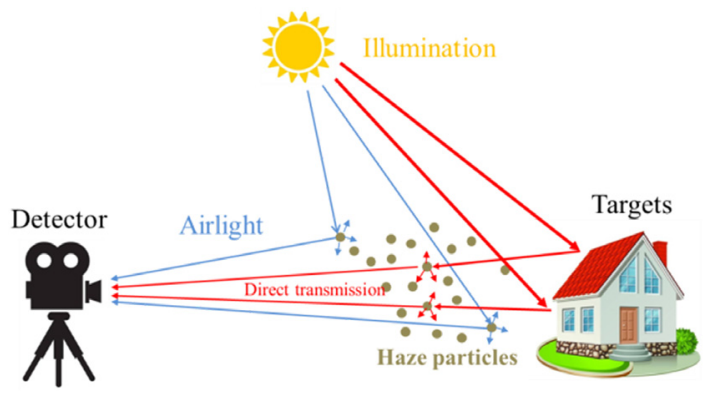

Fig. 2 The schematic of the imaging degradation model in haze., ${ }^{1,2}$ Direct transmission is the object light after attenuation through haze. Airlight is the scattered atmospheric light. ${ }^{1}$ 
the dehazed image is obtained as far as the airlight radiance is estimated and eliminated accurately, and the attenuation of the object light is compensated. By combining Eqs. (1)-(3), we obtain the final dehazed image $L$ as

$$
L(x, y)=\frac{I(x, y)-A(x, y)}{1-A(x, y) / A_{\infty}} .
$$

Since $A_{\infty}$ is a global constant, it can be estimated easily. The key difference among different dehazing methods is the way they estimate the airlight radiance. The airlight that the atmospheric particles scatter is partially linearly polarized, which is determined by Mie scattering theory. ${ }^{42}$ So, the polarimetric dehazing method estimates the airlight radiance using multiple polarization images and focuses on improving the estimation accuracy of the airlight to achieve efficient dehazing results.

\section{Passive Polarimetric Dehazing Methods}

\subsection{Polarimetric Dehazing Methods Based on Polarized-Difference Imaging}

The polarimetric dehazing method was first proposed by Schechner et al. ${ }^{42}$ The authors further reported the details and experiments of this proposed method in $2003 .^{43}$ In this method, the authors design three approximations that are widely used in later methods. First, the authors only consider the degradation caused due to the attenuation of signal and the additive airlight. Second, the authors only regard the scattering as single-scattering effect. Finally, the object light is assumed to be unpolarized. On the basis of the aforementioned approximations, the method proposed in Ref. 42 is designed on the basis of polarized-difference (PD) imaging, i.e., two images with orthometric polarization. When a polarizer is mounted in front of a camera, the total radiance that the camera receives fluctuates with different orientation of the polarizer, resulting from the polarized airlight. This is shown in Fig. 3. The images with the maximum and the minimum radiance correspond to the "worst state" and "best state" and are denoted as $I_{\perp}$ and $I_{\|}$, respectively. The typical "worst state" and "best state" images are shown in Fig. 4. It is evident from the figure that the difference between the two images is very clear. Therefore, there is a need to devise some special methods for the determination of positions, such as subjective

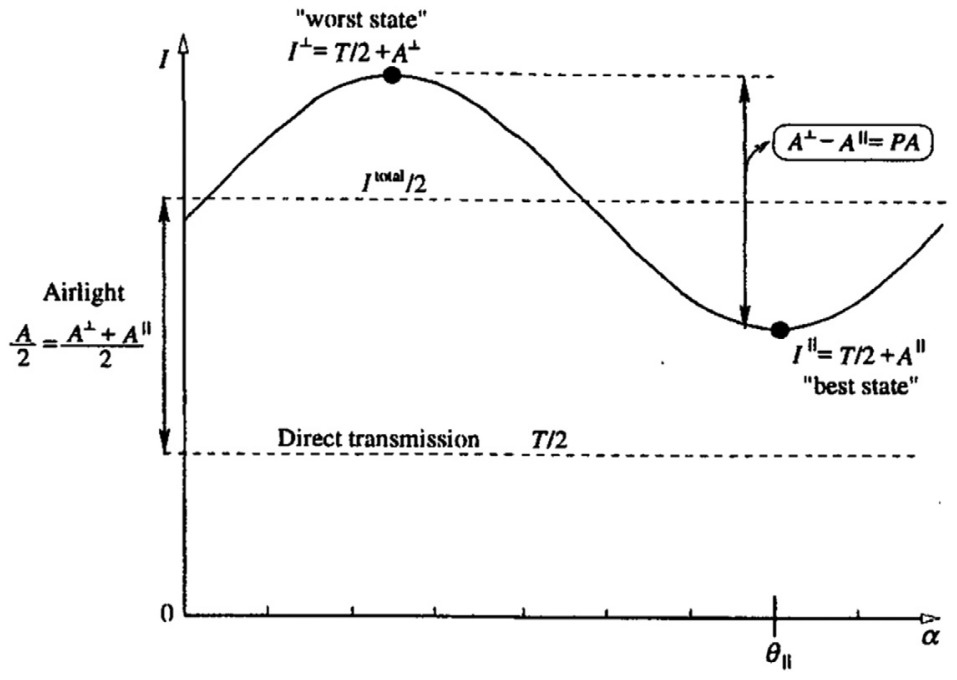

Fig. 3 The relationship between the image radiance and the rotational angle of the polarizer. The measured minimum $I^{\|}$and maximum $I^{\perp}$ intensity are function of $\alpha$. The difference between and is due to the difference between the airlight components $A^{\|}, A^{\perp}$. It is determined to the unknown airlight intensity by the parameter $P_{\mathrm{A}}$. The total intensity $I^{\text {total }}$ is composed of the airlight intensity and the direct transmission. ${ }^{42}$ 


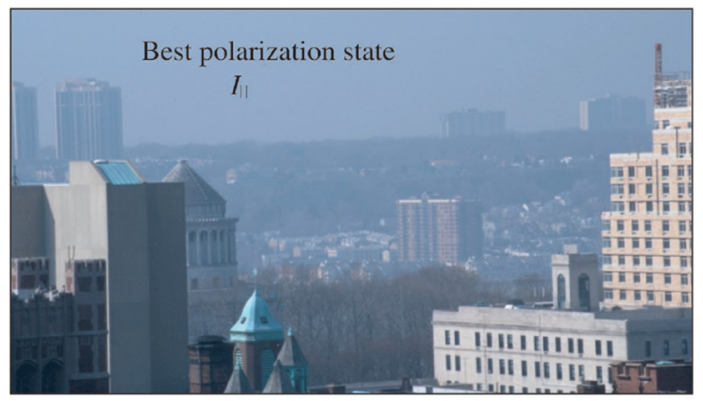

(a)

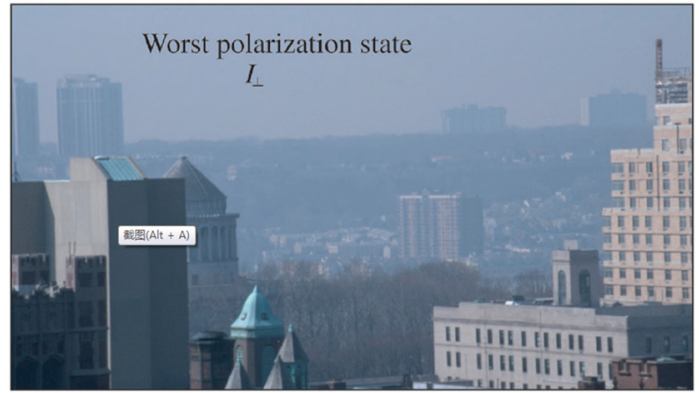

(b)

Fig. 4 Images of the polarization components corresponding to the minimal and the maximal radiances. The "best state" image has the best image contrast and the "worst state" image has the worst image contrast. ${ }^{43}$

judgment, ${ }^{42,43}$ electrically switchable polarizer based on a liquid crystal device, ${ }^{46}$ and Stokes vector deduction. ${ }^{49,67,68}$

We can derive the parameters from the two polarized images. The degree of polarization (DoP) of the airlight is defined as $p_{\mathrm{A}}$ and can be obtained as

$$
P_{A}=\frac{A_{\perp}-A_{\|}}{A_{\perp}+A_{\|}}=\frac{I_{\perp}(\mathrm{sky})-I_{\|}(\mathrm{sky})}{I_{\perp}(\mathrm{sky})+I_{\|}(\mathrm{sky})},
$$

where $I$ (sky) represents the sky area of the image without objects. In this area $I=A$. Thus, the airlight radiance for the whole image is expressed as

$$
A=A_{\perp}+A_{\|}=\frac{A_{\perp}-A_{\|}}{P_{A}}=\frac{I_{\perp}-I_{\|}}{P_{A}} .
$$

The global constant $A_{\infty}$ is obtained by the pixels in area representing the sky as

$$
A_{\infty}=\frac{1}{2}\left[I_{\perp}(\mathrm{sky})+I_{\|}(\mathrm{sky})\right] .
$$

After obtaining the two essential parameters, we obtain the dehazed image as shown in Fig. 5. Meanwhile, the method renders a byproduct, the range map, which indicates the distance ordering of the objects in the scene, with the assumption that the extinction coefficient is distance invariant. The range map is defined as

$$
\beta z=-\ln \left[1-A / A_{\infty}\right]
$$

The range map of Fig. 4 is shown in Fig. 6. It is notable that this map is qualitatively consistent with the scene.

The polarimetric dehazing methods achieve efficient results. However, there are some technical details that still require completion, such as the selection of sky area and the processing for the specular objects. Namer et al. concluded that the sky area close to horizon is more reliable. 


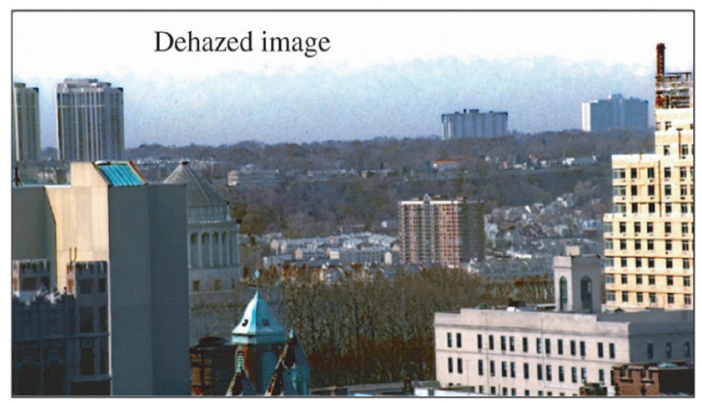

Fig. 5 Dehazing results for Fig. 4. The dehazing image shows much better contrast and color than the hazy images. ${ }^{43}$

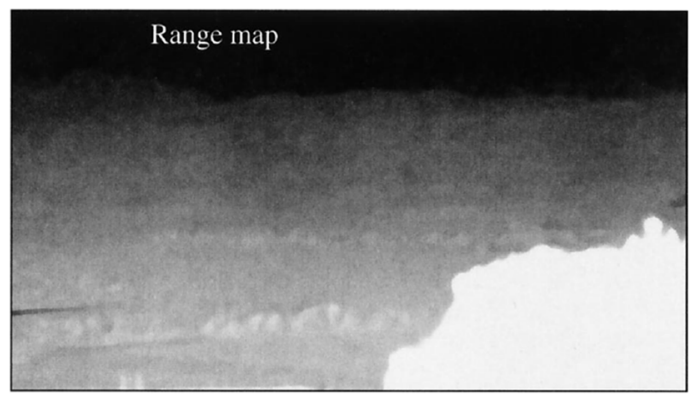

Fig. 6 Range map of the scene for Fig. 4. The farther the object, the darker the shading. ${ }^{43}$

The authors proposed an automatic sky detector. ${ }^{46}$ The sky area detected by the proposed method is shown in Fig. 7(a). Figure 6(b) presents the dehazed image. The cases in which the images do not contain the sky in the field of view are discussed in detail. ${ }^{47,69,70}$ Furthermore, the cases where the third approximation is not satisfied are also deeply analyzed. The dielectric objects, such as water bodies and shiny construction materials, reflect the light toward the camera that is significantly polarized. Such pixels are overcompensated to strange colored pixels or dark pixels. Namer et al. consider that the adjacent objects should show similar airlight value. Once the algorithm automatically detects the areas in the airlight image that are very different from their surroundings, the airlight is automatically re-estimated by simple interpolation of the airlight of the surroundings. Figure 8(a) shows the specular objects in hazy image. The basic method produced black spots in these areas as shown in Fig. 8(b). However, the improved method successfully recovered the colors in these areas as shown in Fig. $8(\mathrm{c}){ }^{46}$ Fang et al. proposed a

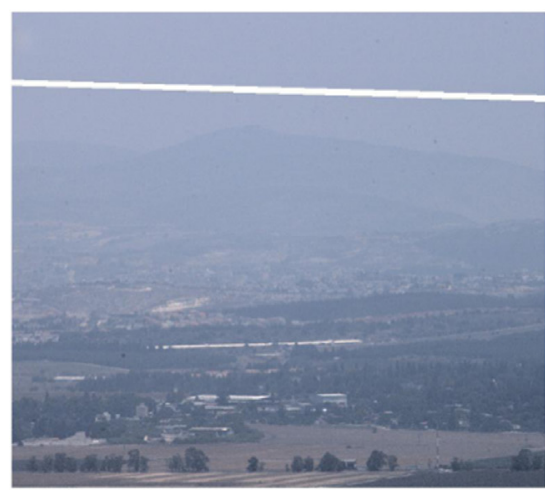

(a)

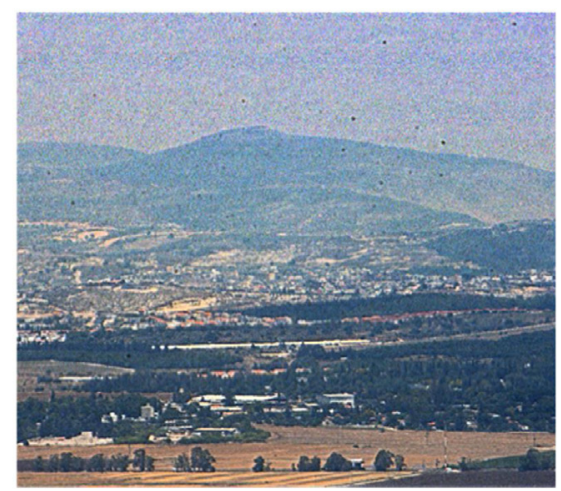

(b)

Fig. 7 (a) The best polarized image $I^{l}$ of a hazy scene. The automatic detected sky is marked by a white line; (b) result of dehazing for scene (a), relying on the automatically selected sky line. ${ }^{46}$ 


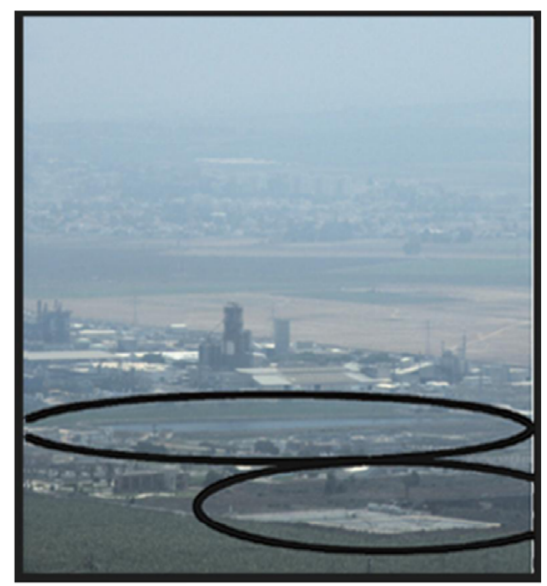

(a)

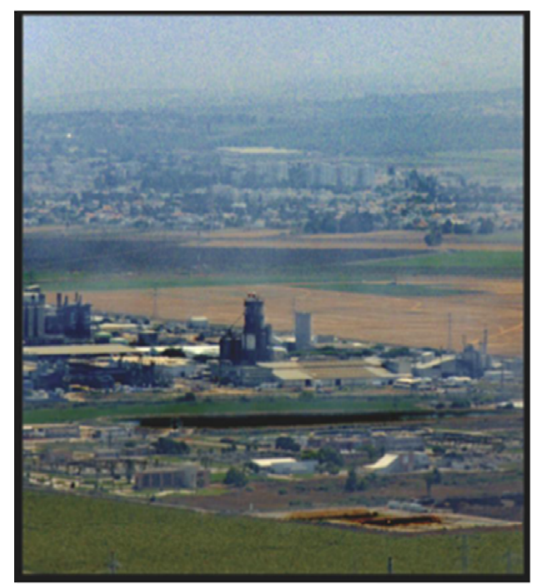

(b)

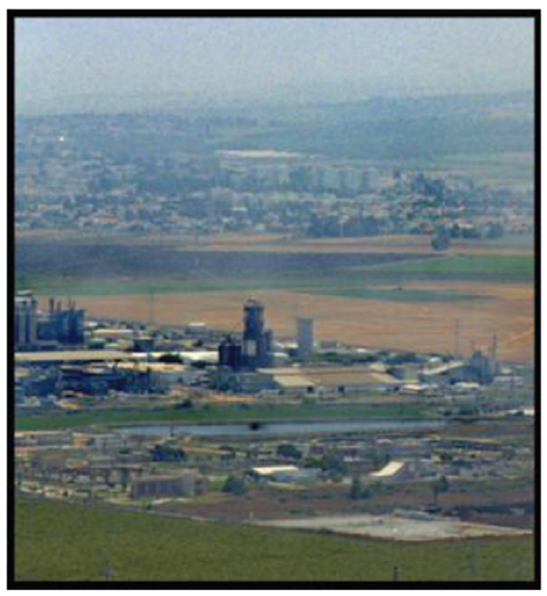

(c)

Fig. 8 Corrected dehazing result when specular objects exist in the field of view. (a) The best polarized hazy image with specular objects; (b) dehazing result without re-estimate the airlight. The color with specular objects area has distorted to black; (c) dehazing result with re-estimate the airlight. The colors are well recovered compared to (b). ${ }^{46}$

decorrelation-based scheme to estimate the DoP for the target objects. Figure 9 shows the results before and after the consideration of the polarization effect of the objects. ${ }^{57}$ Huang et al. proposed a method to estimate the PD image of the signal and search for the best dehazing result in terms of quality. The improved dehazing results are shown in Fig. $10 .^{71}$ In addition, there are various works that discuss the effectiveness of the polarimetric dehazing methods for underwater image quality enhancement. ${ }^{55,56,72-78}$ As shown in Fig. 3, it is found that the two polarized

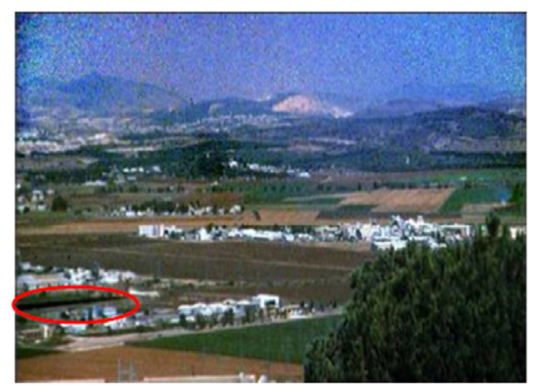

(a)

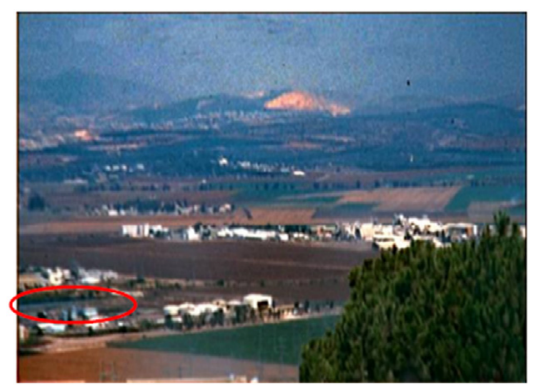

(b)

Fig. 9 (a) Dehazing results using Schechner's method in Ref. 42. (b) Dehazing result with consideration of the polarization effect of the objects using the proposed method in Ref. 57. 


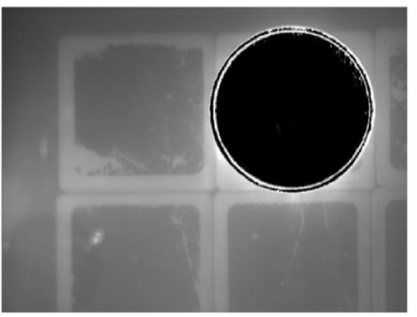

(a)

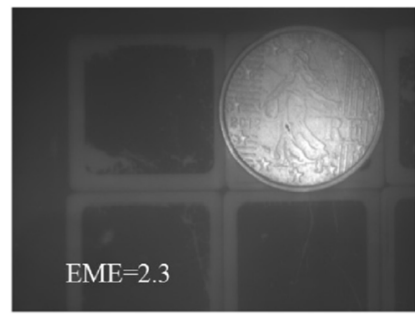

(b)

Fig. 10 The dehazing results (a) before and (b) after the consideration of the polarization effect of the objects in underwater. ${ }^{71}$

images are similar and difficult to distinguish. Thus, the main difficulty for the PD-based method is the acquisition of the "best state" and "worst state" images.

\subsection{Polarimetric Dehazing Methods Based on Stokes Vector}

The PD imaging-based polarimetric dehazing methods easily obtain the DoP of the airlight and achieve effective dehazing results. However, the Stokes vector-based polarimetric dehazing method further obtains the angle of polarization (AoP) of the airlight. This allows to further improve the estimation accuracy of the airlight. So, the Stokes vector-based polarimetric dehazing method has attracted considerable attention. ${ }^{50,51,58-60,79}$ The Stokes vector comprises four parameters. It represents the polarization property of light on the basis of the intensity information, which makes the representation and detection of polarized light much easier ${ }^{80}$ In the dehazing field, we generally consider that the airlight is related to the linear polarization effect, i.e., only the first three parameters. In order to obtain the linear Stokes vector, three $(0 \mathrm{deg}, 60 \mathrm{deg}$, and $120 \mathrm{deg})^{79}$ or four $(0 \mathrm{deg}, 45 \mathrm{deg}, 90 \mathrm{deg} \text {, and } 135 \mathrm{deg})^{50,51}$ images with different polarization orientations are required. Liang et al. adopted the four images captured by the polarizer at orientations of $0 \mathrm{deg}, 45 \mathrm{deg}, 90 \mathrm{deg}$, and $135 \mathrm{deg}$, for extracting the DoP and AoP of the airlight. ${ }^{50,51}$ The intensities of the four images are $I(0), I(45), I(90)$, and $I(135)$. This is shown in Fig. 11. The linear Stokes vector is

$$
\left\{\begin{array}{l}
S_{0}=I(0)+I(90) \\
S_{1}=I(0)-I(90) \\
S_{2}=I(45)-I(135)
\end{array},\right.
$$

where $S_{0}$ denotes total radiance, i.e., $I, S_{1}$ denotes the intensity difference between the vertical and horizontal polarized components, and $S_{2}$ denotes the intensity difference between the $45 \mathrm{deg}$ and 135 deg polarized components with respect to $x$ axis.

According to the definition of the Stokes vector, the DoP and AoP are obtained as

$$
p=\frac{\sqrt{S_{1}^{2}+S_{2}^{2}}}{S_{0}} \quad \theta=\frac{1}{2} \arctan \left[\frac{S_{2}}{S_{1}}\right] .
$$

Based on the discussion in Sec. 3.1, it is evident that $S_{0}$ includes the direct transmission, but $S_{1}$ and $S_{2}$ so not include it. Thus, the DoP is influenced by the direct transmission, whereas the AoP is not affected by it. Consequently, the estimation of the airlight is more accurate using AoP as compared to DoP. The AoP that exists in the highest frequency in the whole image is defined as the AoP of the airlight $\left(\theta_{\mathrm{A}}\right)$. Now, the DoP of the airlight $\left(p_{\mathrm{A}}\right)$ is defined as the maximum value among the pixels that satisfies $\theta_{\mathrm{A}}$. For simplicity, the directions of 0 deg and $90 \mathrm{deg}$ are defined as $x$ and $y$ axes, respectively. Thus, $\theta_{\mathrm{A}}$ represents the angle between the polarization orientation of the airlight and $x$ axis. $A_{\mathrm{p}}$ denotes the polarized radiance of the airlight. In this case, the polarized radiance of the airlight in $x$ and $y$ directions is expressed as $A_{p x}=A_{\mathrm{p}} \cdot \cos ^{2} \theta_{\mathrm{A}}$ and $A_{p y}=A_{\mathrm{p}} \cdot \sin ^{2} \theta_{\mathrm{A}}$. Considering the fact that $A_{\mathrm{px}}$ and $A_{\mathrm{py}}$ are also mathematically expressed as 


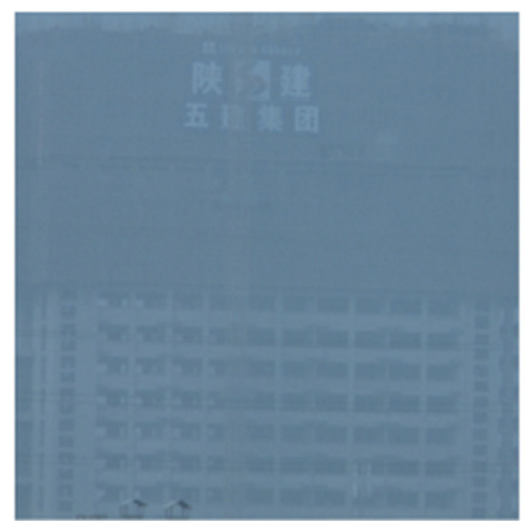

(a)

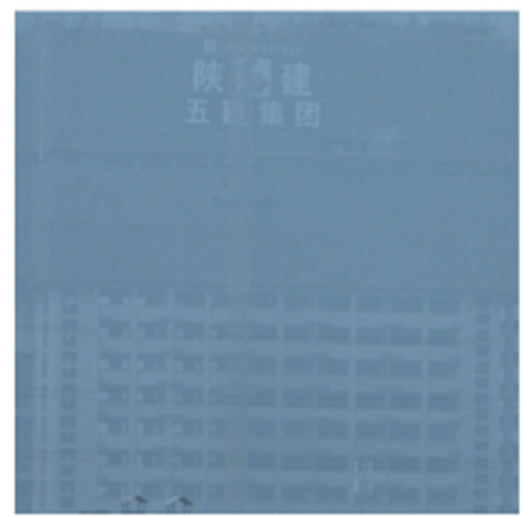

(c)

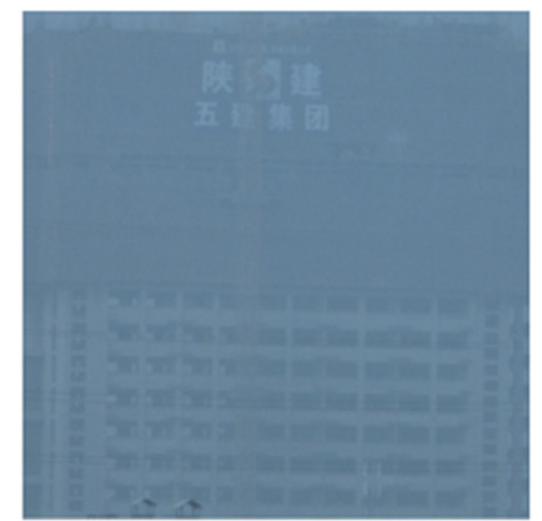

(b)

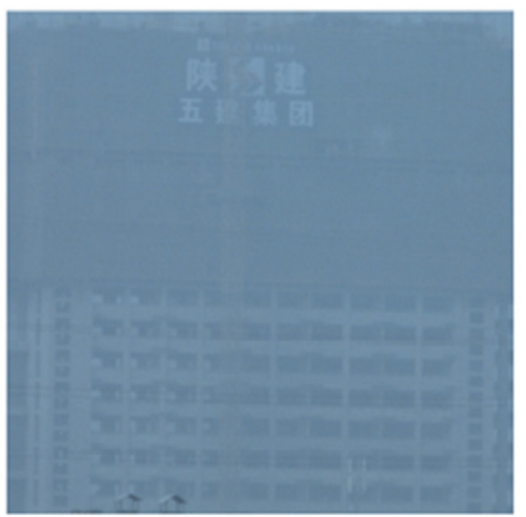

(d)

Fig. 11 The hazy images with the polarizer at the orientations of (a) 0 deg, (b) 45, (c) 90 deg, and (d) 135 deg, respectively. ${ }^{51}$

$$
A_{p x}=I_{0}-S_{0}[1-p] / 2 \quad A_{p y}=I_{90}-S_{0}[1-p] / 2
$$

we establish the following relation:

$$
A_{p}=\frac{I_{0}-S_{0}[1-p] / 2}{\cos ^{2} \theta_{A}}=\frac{I_{90}-S_{0}[1-p] / 2}{\sin ^{2} \theta_{A}}
$$

Now, $A$ is easily obtained by using $A=A_{\mathrm{p}} / p_{\mathrm{A}}$.

In order to cater the sky area, the authors proposed a new method for the estimation of $A_{\infty}$. The proposed method is effective and accurate for almost all conditions. The final dehazing result is shown in Fig. 12.

Liang et al. also proposed a scheme for improving the performance of the polarimetric dehazing methods in dense haze condition. ${ }^{81}$ It is inferred that the quantization error of the camera is a major problem in dense haze conditions. As a result, the noise is also significantly amplified. In order to eliminate the influence of quantization noise, the local average filter is employed in a small patch. Figure 13 shows the AoP distribution before and after the optimization. It is evident that the results are more accurate after optimization. Figure 14 shows the dehazing results using the optimized method and the basic method. ${ }^{51}$ The dehazing capacity of the optimized polarimetric dehazing methods is effectively improved. In Ref. 82, the authors compared the experimental performances of the polarimetric dehazing method based on three random angles and two orthogonal angles. The experimental results show that the three random angles-based method outperforms the two orthogonal angles-based method. Moreover, the former method does not require accurate angle, thus making this method more feasible for other applications. 


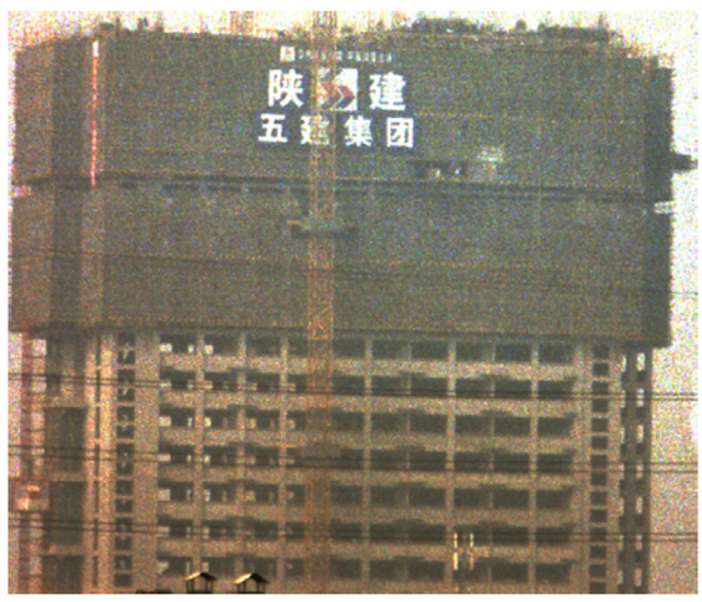

Fig. 12 Dehazing result dealt with the proposed method in Ref. 51.

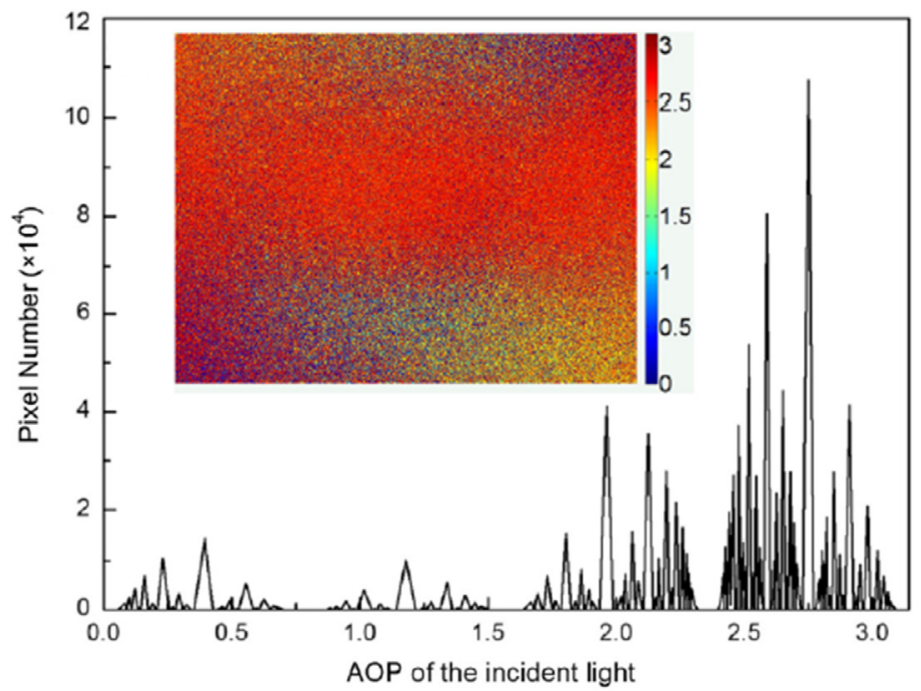

(a)

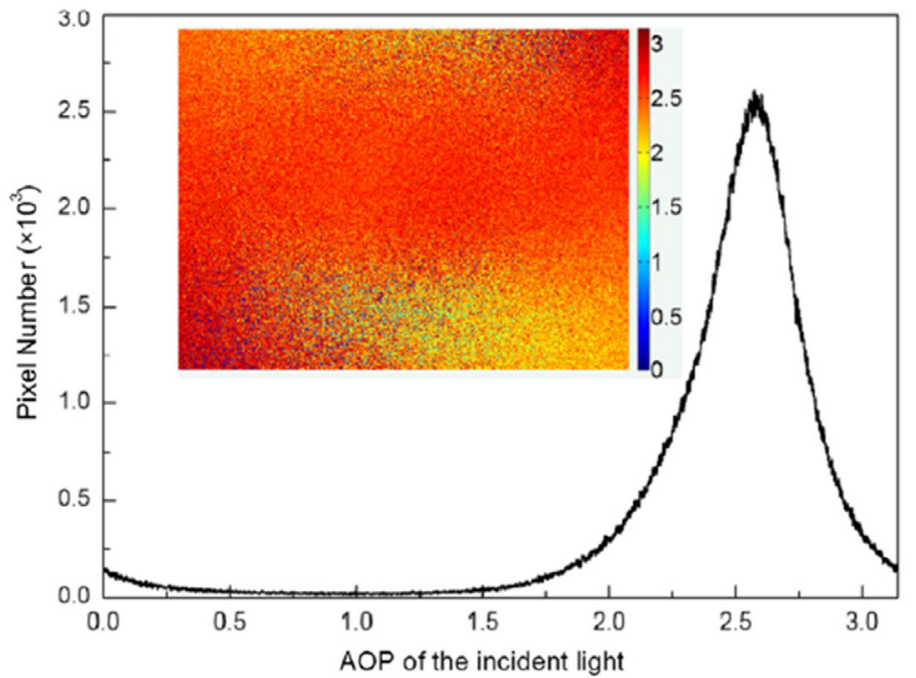

(b)

Fig. 13 (a) The AoP value of the incident light before optimization. (b) The AoP value of the incident light after smoothing each pixel value of the four raw images. ${ }^{81}$ 


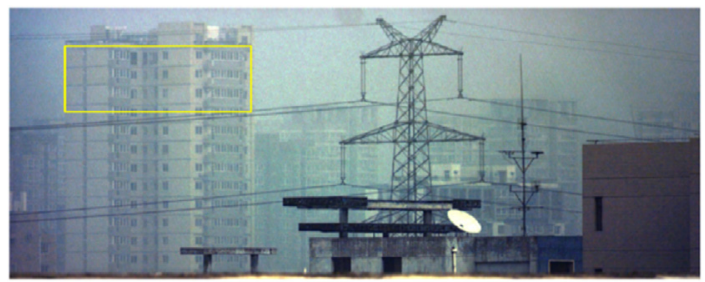

(a)

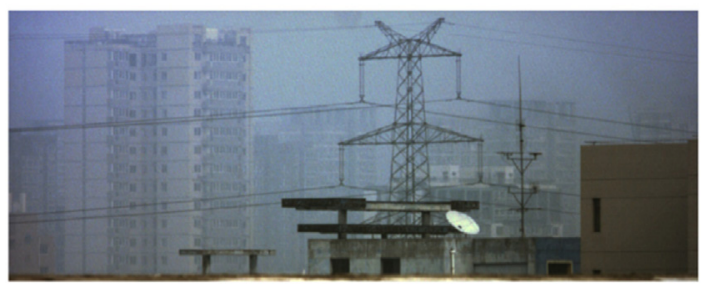

(b)

Fig. 14 Dehazing results using (a) the optimized polarimetric dehazing method in Ref. 81 and (b) the basic polarimetric dehzing method in Ref. 51.

\subsection{Polarimetric Dehazing Methods Incorporating Digital Image Processing}

Image enhancement is a basic and effective technique in computer vision. Various image enhancement algorithms can be designed into the polarimetric dehazing method to improve the dehazing capacity. Liu et al. considered that in hazy images, the objects and haze differ in spatial frequency distribution. The low and high spatial frequency components reflect the effects of haze and objects, respectively. In the method proposed by Liu et al., the hazy image is decomposed into different spatial frequency layers using the wavelet transform. First, the low spatial frequency components are processed with the polarimetric dehazing method. Then, the high spatial frequency components are manipulated with a nonlinear transform. ${ }^{64,83}$ The corresponding dehazing results are shown in Fig. 15. There are other works presented in literature that have made efforts in making a synergy of digital image processing algorithms and polarimetric dehazing methods. ${ }^{84-87}$ The main obstacle for this method is the algorithm complexity. More effort should be made for real-time application.

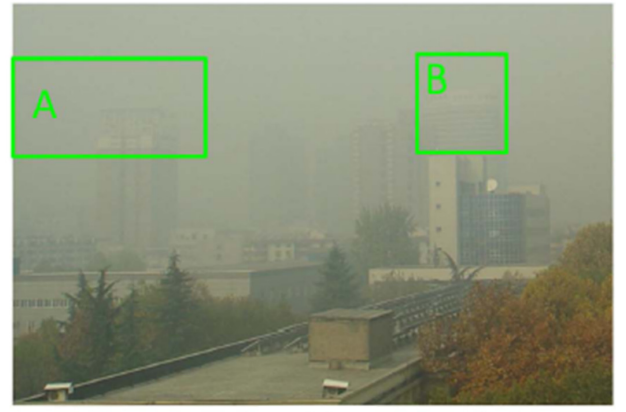

(a)

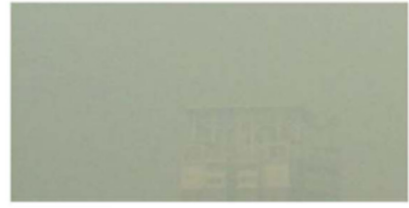

(c)

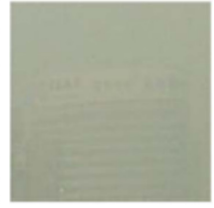

(d)

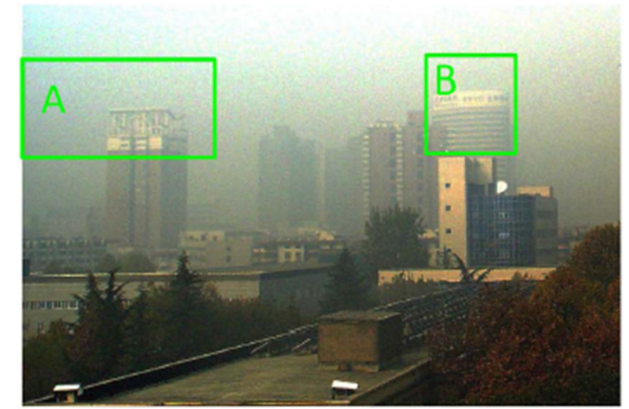

(b)

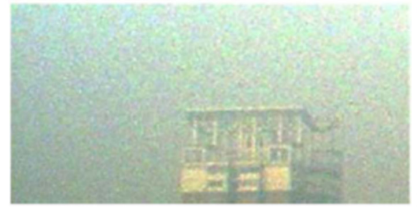

(e)

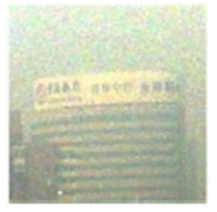

(f)

Fig. 15 Dehazing results. (a) Hazy image; (b) dehazing results with the proposed method; (c) magnified region on the green rectangle $A$ in (a); (d) magnified region on the green rectangle $B$ in (a); (e) magnified region on the green rectangle $A$ in (b); and (f) magnified region on the green rectangle $B$ in (b). ${ }^{68}$ 


\subsection{Polarimetric Dehazing Methods Based on Visible and Infrared Image Fusion}

In general, the haze (including mist) particle size is almost $<1 \mu \mathrm{m}$, where the light propagation obeys Mie scattering theory. The near-infrared light propagates to a greater distance as compared to visible light due to low scattering. An image of good quality is obtained by combining the color information of visible image and the high visibility of the near-infrared image. However, the quality of the near-infrared hazy images can also be improved after the processing the image using the polarimetric dehazing method. ${ }^{49}$ Liang et al. proposed the polarimetric dehazing method that fuses the visible dehazed image and near-infrared dehazed image to advance the visibility. ${ }^{88}$ This dehazing process for a color hazy image consists of two steps: (1) applying the basic polarimetric dehazing method to visible and near-infrared hazy images separately; (2) combining the visible and near-infrared dehazed images to obtain the final dehazed image using a fusion algorithm. Figure 16 shows the original groups of visible and near-infrared hazy images. As presented in the figure, the near-infrared image inherently contains more information and better visibility as compared to the normal image. This is consistent with the discussions presented in Refs. 39 and 40.

The visible and near-infrared dehazed images are processed by the basic polarimetric dehazing method ${ }^{51}$ as presented in Fig. 17. It is evident that the contrast of the visible and near-infrared dehazed images is enhanced significantly as compared to the images presented in Figs. 16(a) and 16(b). In addition, the resultant image obtained by fusing the visible and near-infrared dehazed images are enhanced and the color information is also restored. This is shown in Fig. 18(b). On the other hand, the resultant image of directly fused visible and near-infrared hazy images is shown in Fig. 18(a), which can be roughly regarded as the visible and near-infrared fusion

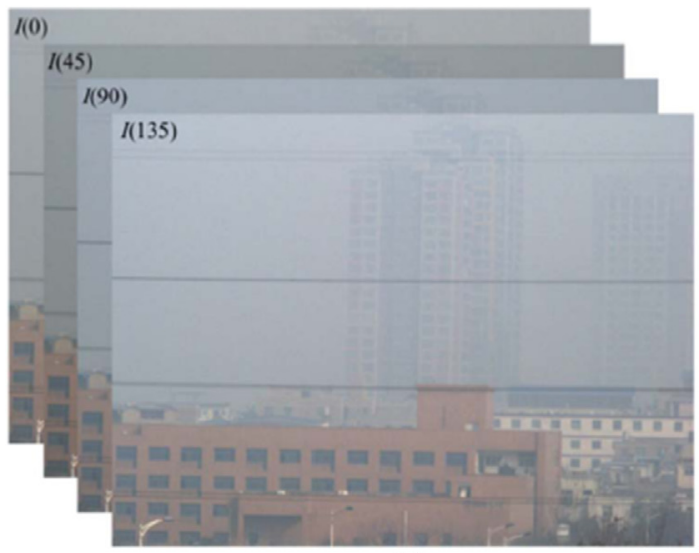

(a)

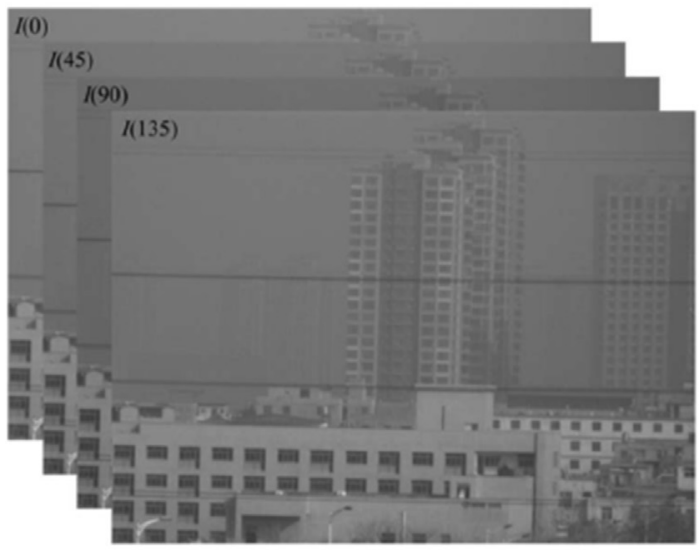

(b)

Fig. 16 Original groups of (a) visible hazy images and (b) near-infrared hazy images. ${ }^{88}$ 


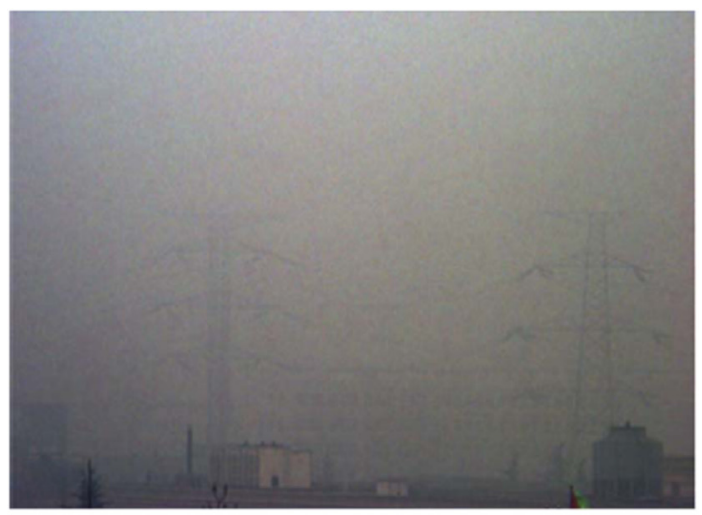

(a)

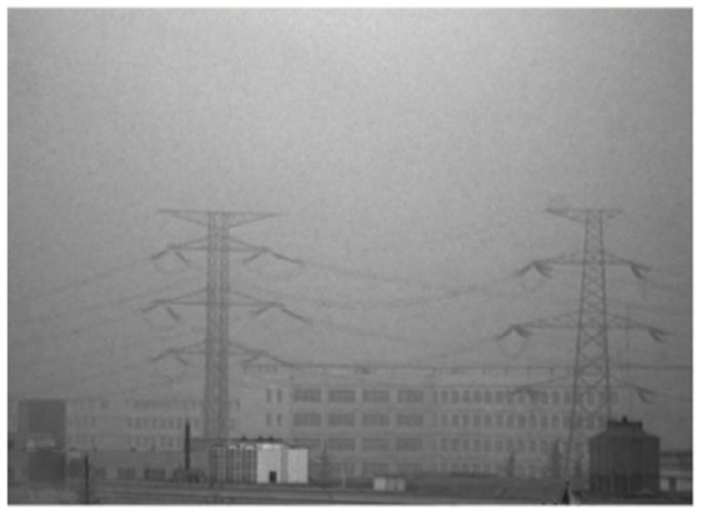

(b)

Fig. 17 Dehazed images of Fig. 16 by polarimetric dehazing method. (a) Dehazed visible image with color; (b) dehazed near-infrared image. ${ }^{88}$

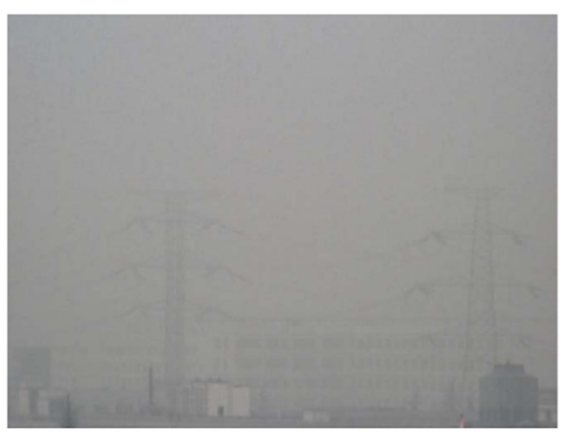

(a)

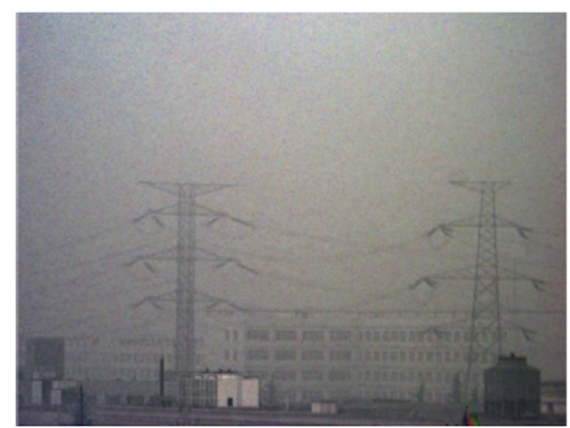

(b)

Fig. 18 (a) Fused image of visible and near-infrared hazy image. (b) Fused image of visible and near-infrared dehazed image, i.e., the final dehazing result. ${ }^{88}$

method. ${ }^{50,51}$ The process of directly fusing the hazy images together is unable to enhance the quality of hazy images in dense haze conditions. The experimental results demonstrate that the visibility of the final dehazed image is improved at least $100 \%$. Two shortcomings of the proposed method limit its further application in dehazing field. On the one hand, the simultaneous acquisition of the visible and near-infrared images with the same scenes. It usually needs additional image registration to make them consistent. On the other hand, the computational complexity requires several hours for processing an image of 100 megapixels. 


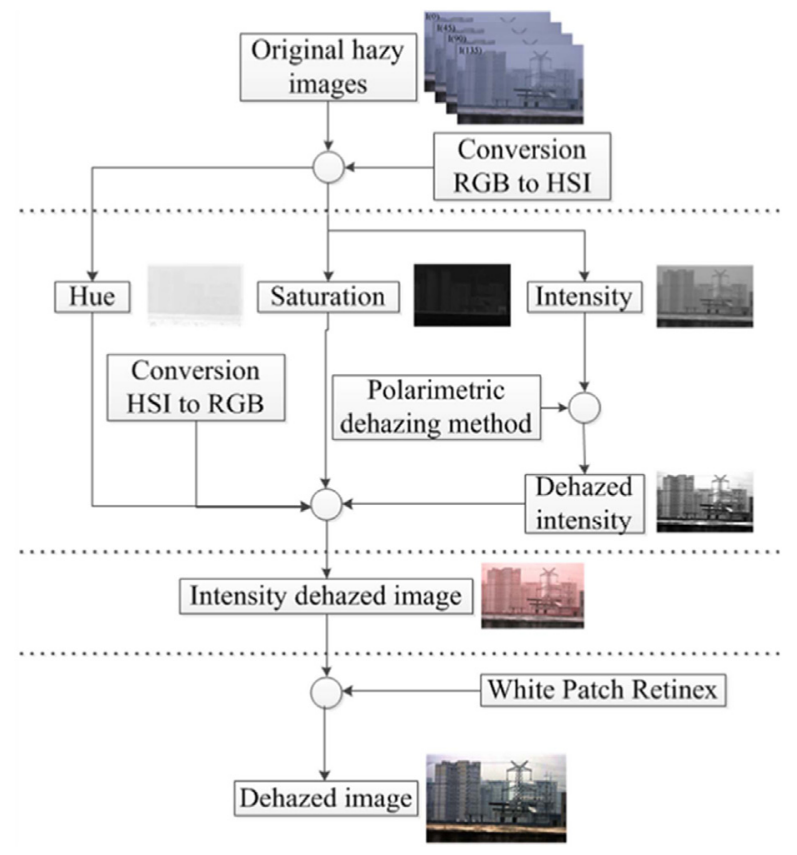

Fig. 19 The overall flowchart of the proposed method. ${ }^{89}$

\subsection{Fast Polarimetric Dehazing Method}

Many practical applications, such as the traffic monitoring and navigation system, require realtime processing. In addition to the image acquisition systems, the efficiency of the polarimetric dehazing method is of significance for the real-time image dehazing. Zhang et al. proposed a fast polarimetric dehazing method in HSI color space and for color correction. ${ }^{89}$ In HSI color space, the intensity channel is only related to the RGB intensities. ${ }^{90}$ So, the polarimetric dehazing process is only implemented once in the intensity channel. The color distortions, which result from different scattering coefficients, are dependent on the wavelengths and are corrected by the white patch retinex method. The overall flowchart of the proposed method is shown in Fig. 19. The experiments indicate that the quality of the dehazed image obtained using the proposed method is similar to that of the polarimetric dehazing method in RGB color space. Table 1 shows the execution time of different methods. The proposed method outperforms Tarel in terms of efficiency, which is developed as a fast-dehazing method and shows the advantage in computational efficiency. ${ }^{89}$

\subsection{Real-Time Polarimetric Dehazing Method Based on Imaging Polarimeter}

In order to perform the true real-time image haze removal, the real-time image acquisition and real-time processing should be guaranteed. In case of polarimetric dehazing method, the simultaneous acquisition of polarization images relies on the imaging polarimeter. The imaging polarimeter is a device that can obtain four different linear polarization images simultaneously

Table 1 The consuming time of the different methods. ${ }^{89}$

\begin{tabular}{lcccccc}
\hline \hline Image size $(h * w)$ & Meng & MSRCR & Tarel & HE & RGB PDM & Work in Ref. 89 \\
\hline $727^{*} 1150$ & $10.78 \mathrm{~s}$ & $7.77 \mathrm{~s}$ & $35.48 \mathrm{~s}$ & $1.24 \mathrm{~s}$ & $86.27 \mathrm{~s}$ & $30.99 \mathrm{~s}$ \\
$950 * 1300$ & $11.52 \mathrm{~s}$ & $11.02 \mathrm{~s}$ & $68.85 \mathrm{~s}$ & $1.27 \mathrm{~s}$ & $140.31 \mathrm{~s}$ & $52.91 \mathrm{~s}$ \\
$970 * 1300$ & $11.57 \mathrm{~s}$ & $11.53 \mathrm{~s}$ & $69.71 \mathrm{~s}$ & $1.36 \mathrm{~s}$ & $142.53 \mathrm{~s}$ & $52.96 \mathrm{~s}$ \\
$690 * 1180$ & $8.29 \mathrm{~s}$ & $6.90 \mathrm{~s}$ & $38.98 \mathrm{~s}$ & $1.11 \mathrm{~s}$ & $88.12 \mathrm{~s}$ & $31.27 \mathrm{~s}$ \\
\hline \hline
\end{tabular}




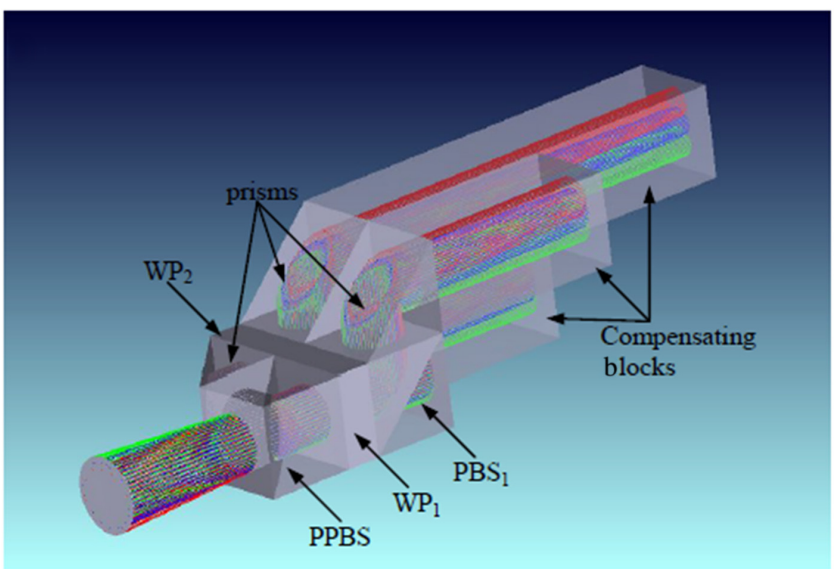

(a)

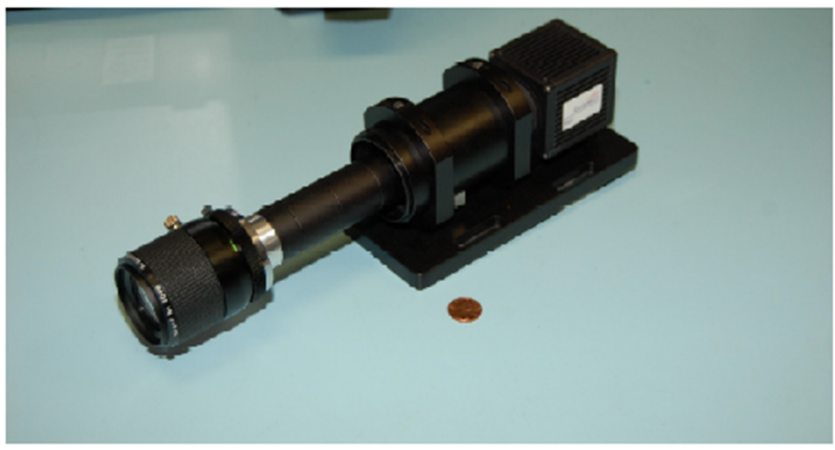

(b)

Fig. 20 (a) The optical construction and (b) the prototype of the division of amplitude near-infrared polarimeter. ${ }^{73}$

(linear-Stokes polarimeter) or three linear polarization images and one circular polarization image (full-Stokes polarimeter) with optimal designed structures. ${ }^{91}$ The polarimeters are usually based on different technological aspects, such as division of time, ${ }^{92-95}$ division of amplitude, ${ }^{96}$ division of aperture, ${ }^{60,97}$ division of focal plane, ${ }^{98-100}$ and Fourier-based. ${ }^{101-104}$ The imaging polarimeter has been thoroughly reviewed in Ref. 91, thereby we only focus on the development on polarimeters related to image dehazing. Mudge et al. presented the real-time dehazing results based on a division of amplitude near-infrared polarimeter. ${ }^{49,96}$ The optical construction is shown in Fig. 20(a). The four channels focus on one sensor with the resolution of $640 * 512$. The prototype is shown in Fig. 20(b). Figure 21(a) shows the hazy image provided by the polarimeter, and the quality of the dehazed image is shown in Fig. 21(b). The resultant figure shows that the quality of the image is improved. However, the prototype is controlled by a laptop computer using LabVIEW, and MATLAB is used to process and display the polarimetric dehazing images, which is not real-time dehazing in true sense.

Zhang et al. proposed a true real-time polarimetric dehazing visible polarimeter. ${ }^{60}$ The polarimeter is based on the division of aperture structure and four optical channels, which measure the full-Stokes parameters and focus on one sensor with resolution of $2048 * 2048$. Figure 22(a) shows the polarization-state distribution on the sensor, and the photo of the prototype is shown in Fig. 22(b). Theoretically, the four subimages are uniform with resolution of $1024 * 1024$. However, due to the misregistration in four optical channels, shown in Fig. 23, the polarimeter must be precisely calibrated to mitigate the mismatch of angles of the polarizers and the three linear polarization images in intensity. Figure 24 shows the final dehazed image provided by the polarimeter. It is notable that the quality of the image is significantly improved, and the color information is recovered as well. The dehazing algorithm is loaded into the FPGA modules assembled in the polarimeter that processes the images automatically at a rate of $25 \mathrm{fps}$. It only needs power supply to operate and a monitor to display the dehazed image. 


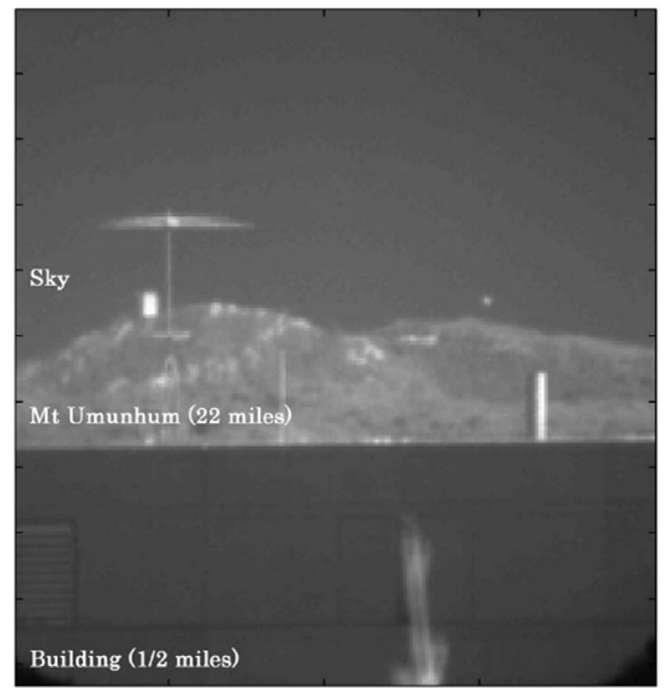

(a)

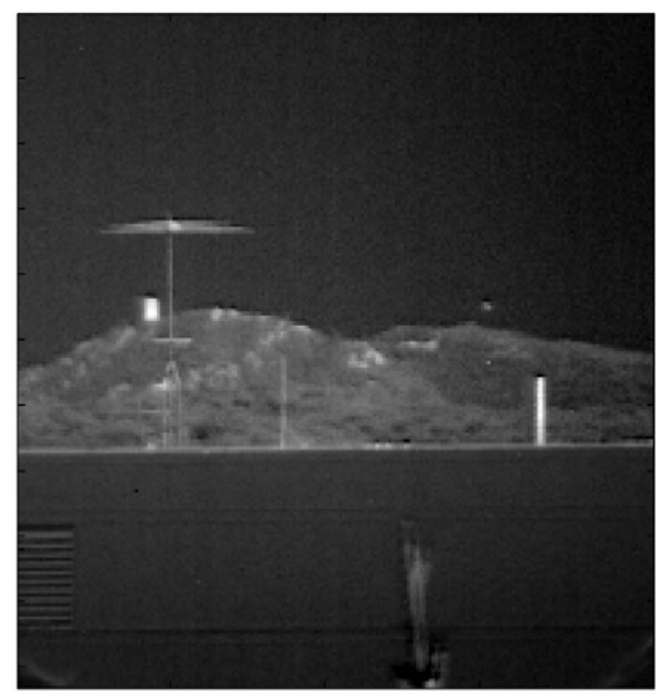

(b)

Fig. 21 (a) The hazy image provided by the polarimeter; (b) dehazed image automatically dehazed by the polarimeter. ${ }^{49}$

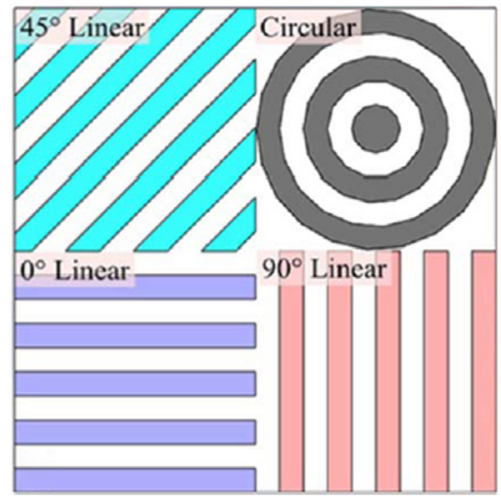

(a)

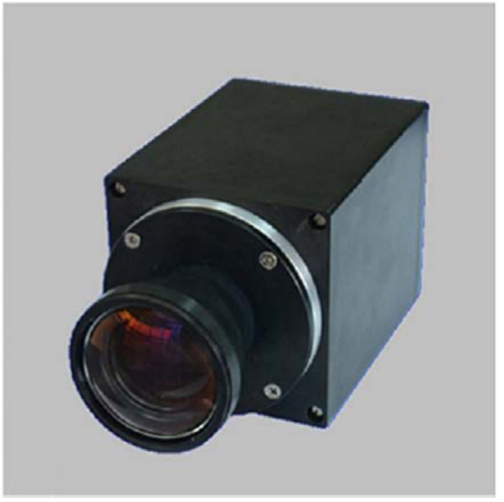

(b)

Fig. 22 (a) The distribution of the polarization-state on the sensor. (b) The photo of the full Stokes polarimetric camera. ${ }^{60}$

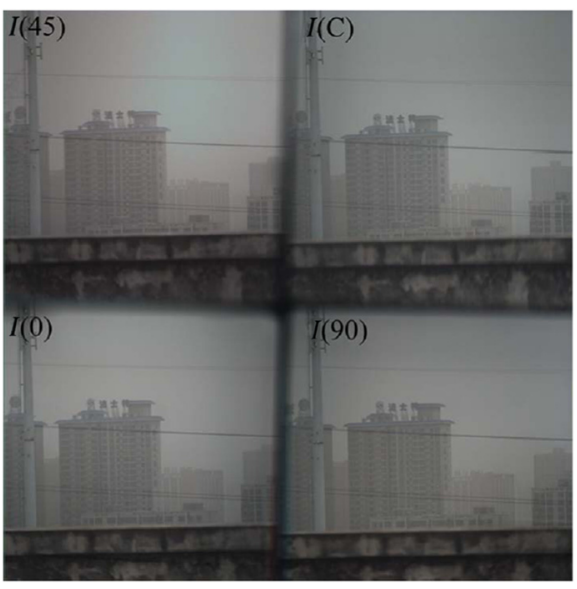

Fig. 23 The original image obtained on single sensor directly without any additional processing. It contains four polarized images, including three linearly, and one circularly polarized images. ${ }^{60}$ 


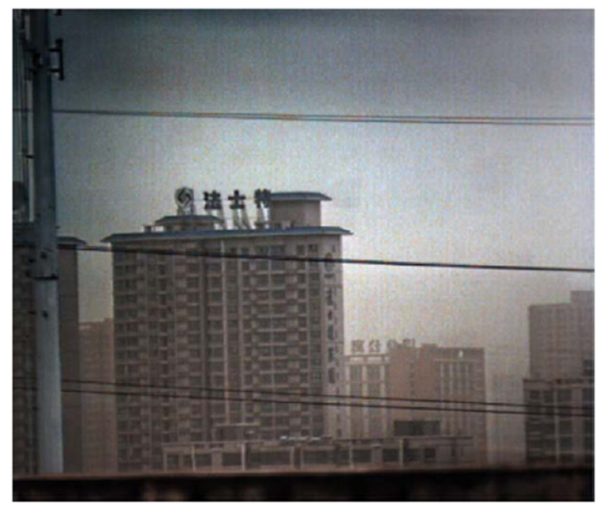

Fig. 24 The final dehazed image dealt with the polarimeter. ${ }^{60}$

\section{Comparison Study for Various Dehazing Methods}

\subsection{Experimental Results}

In this section, we perform some experiments to compare the dehazing capacity of the polarimetric dehazing methods and classic single image dehazing methods. The single image dehazing methods include He's dark channel prior (DCP), ${ }^{23}$ Meng's method (Meng), ${ }^{26}$ Rahman's multiscale retinex for color restoration (MSRCR) ${ }^{8}$ Tarel's method (Tarel) ${ }^{21}$ and Cai's deep learning dehazing method (DehazeNet). ${ }^{33}$ The polarimetric dehazing methods utilize the method in RGB color space (RGB PDM) ${ }^{59}$ and in HSI color space (HSI PDM) ${ }^{89}$ Other reviews of image dehazing methods only compare the performance of some classic single image dehazing methods, ${ }^{34,105}$ this is because they are difficult to obtain the polarized images and the results handle by polarimetric dehazing methods. In our experiments, the original hazy images are captured and the dehazed results are handled by ourselves for all methods. The image dehazing results are shown in Figs. 25-27 for scenes 1 to 3, respectively.

\subsection{Objective Evaluation}

From subjective evaluation, it is obvious that all the image qualities are all improved after dehazing. The visibility of dehazed results handled by polarimetric dehazing methods is better than that of single image dehazing methods. Tarel's method gives rise to color distortion. The Meng's method may obtain the best visibility among the single image dehazing methods.

Objective evaluation should be employed to objectively assess the image quality after dehazing. The quality evaluation of dehazed image is another intense research field, especially for the

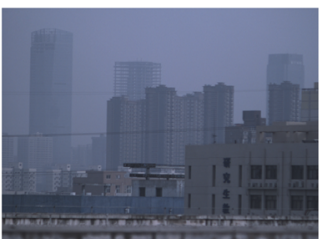

(a)

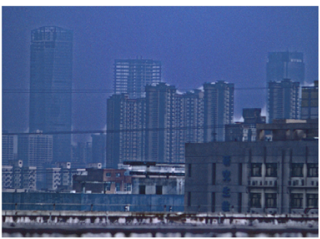

(e)

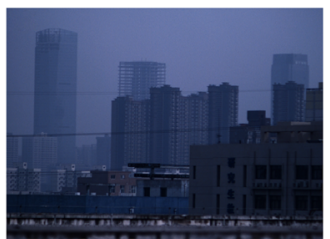

(b)

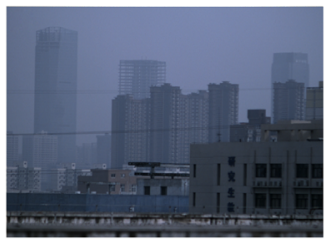

(f)

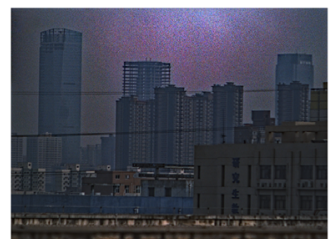

(c)

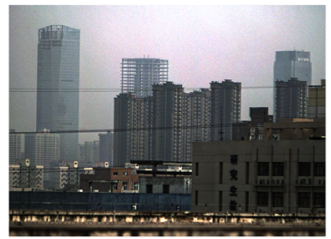

(

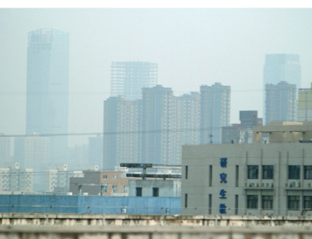

(d)

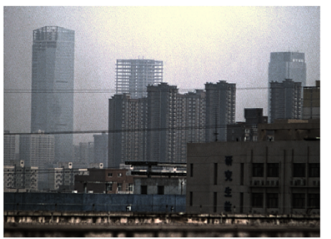

(h)

Fig. 25 Comparisons of some classic dehazing methods with scene 1. (a) Hazy image; (b) DCP; (c) Meng; (d) MSRCR; (e) Tarel; (f) DehazeNet; (g) RGB PDM; and (h) HSI PDM. 


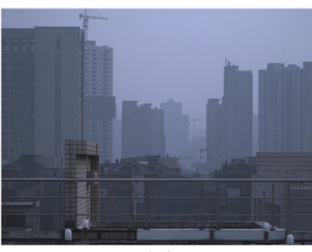

(a)

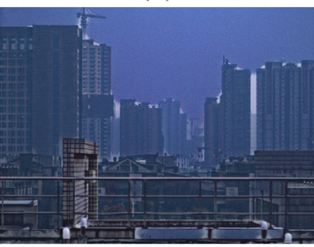

(e)

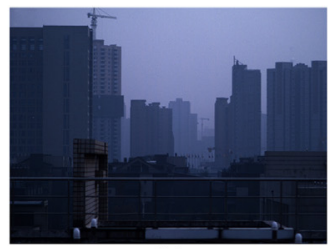

(b)

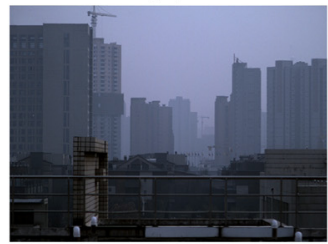

(f)

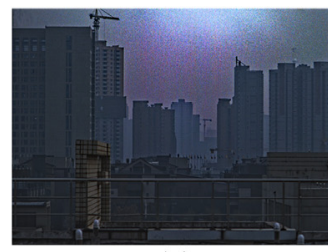

(c)

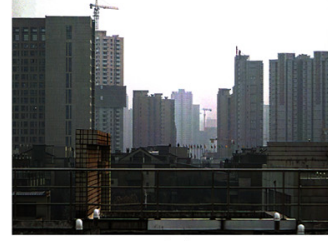

(g)

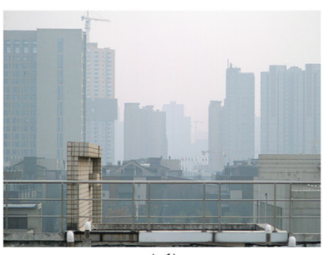

(d)

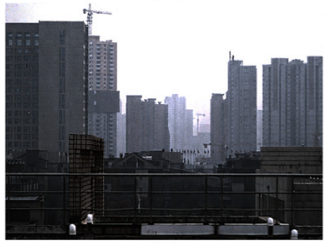

(h)

Fig. 26 Comparisons of some classic dehazing methods with scene 2. (a) Hazy image, (b) DCP, (c) Meng, (d) MSRCR, (e) Tarel, (f) DehazeNet, (g) RGB PDM, and (h) HSI PDM.

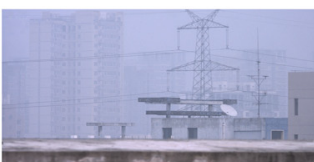

(a)

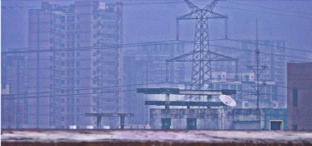

(e)

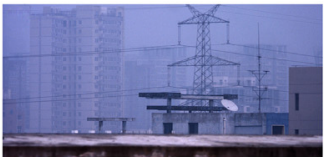

(b)

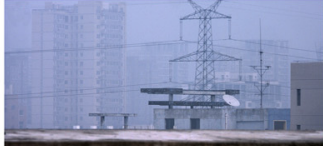

(f)

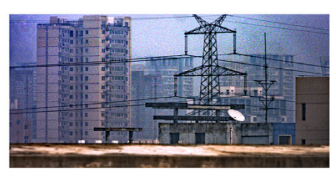

(c)

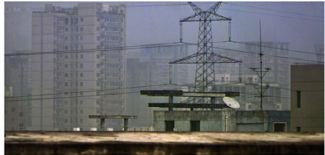

(g)

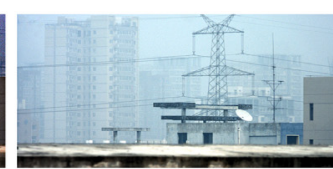

(d)

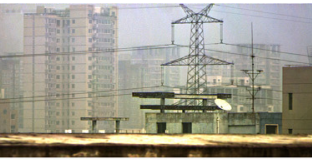

(h)

Fig. 27 Comparisons of some classic dehazing methods with scene 3. (a) Hazy image, (b) DCP, (c) Meng, (d) MSRCR, (e) Tarel, (f) DehazeNet, (g) RGB PDM, and (h) HSI PDM.

situation that the ground-truth haze-free image is not available, i.e., the no-reference image quality assessment (NR IQA). Some NR IQAs have been proposed for objective evaluation, such as the first two indicators $(e, \bar{r})$ of the blind assessment, ${ }^{106}$ image visibility measurement (IVM), ${ }^{107}$ image contrast, ${ }^{108}$ visual contrast (VCM), ${ }^{109}$ natural image quality evaluator (NIQE), ${ }^{110}$ image structure similarity (SSIM), and universal quality index (UQI). ${ }^{111}$ However, few assessments can assess the overall dehazing quality. These eight objective quality evaluation indexes were used to

Table 2 The objective image quality comparison of dehazing results of Fig. 25 with different evaluation indexes.

\begin{tabular}{lccccccc}
\hline \hline Quality evaluation & DCP & Meng & MSRCR & Tarel & DehazeNet & RGB PDM & HSI PDM \\
\hline$e$ & 15.1843 & 36.6753 & 2.4049 & 8.3691 & 5.9937 & $\mathbf{3 8 . 0 8 5 1}$ & 32.1501 \\
$\bar{r}$ & 1.1097 & 2.8316 & 2.6109 & 2.7721 & 1.2186 & $\mathbf{3 . 4 5 9 5}$ & 3.142 \\
IVM & 4.4097 & $\mathbf{1 0 . 2 0 1 5}$ & 1.4675 & 2.8339 & 2.2071 & 9.6982 & 8.4591 \\
Contrast gain & 0.0885 & 0.3024 & 0.0445 & 0.1216 & 0.0515 & $\mathbf{0 . 3 0 7 3}$ & 0.2648 \\
VCM & 39.3762 & 25.731 & 28.0707 & 28.2651 & $\mathbf{3 9 . 5 7 1 2}$ & 37.4269 & 32.3587 \\
NIQE & & & & & & & \\
SSIM $^{a}$ & 8.0489 & 6.8863 & 5.7373 & 6.4582 & 7.2732 & 4.9634 & $\mathbf{4 . 0 9 4 8}$ \\
UQI $^{\mathrm{a}}$ & 0.8326 & 0.7702 & 0.776 & 0.8212 & 0.9654 & 0.7177 & $\mathbf{0 . 7 1 6}$ \\
\hline \hline
\end{tabular}

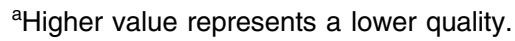


Table 3 The objective image quality comparison of dehazing results of Fig. 26 with different evaluation indexes.

\begin{tabular}{lccccccc}
\hline \hline Quality evaluation & DCP & Meng & MSRCR & Tarel & DehazeNet & RGB PDM & HSI PDM \\
\hline$e$ & 10.8615 & 26.0831 & 0.2466 & 0.8098 & 9.391 & $\mathbf{2 7 . 1 4 7 3}$ & 26.1381 \\
$\bar{r}$ & 0.8459 & 2.3314 & 2.2104 & $\mathbf{5 . 8 0 1 6}$ & 1.2342 & 2.492 & 2.4632 \\
IVM & 4.438 & 8.9944 & 1.9363 & 6.1408 & 4.0719 & $\mathbf{1 0 . 7 5 5 5}$ & 10.6554 \\
Contrast gain & 0.0892 & 0.237 & 0.0271 & 0.2854 & 0.1593 & $\mathbf{0 . 3 1 6 6}$ & 0.3096 \\
VCM & 34.6154 & 23.8866 & 53.2389 & 30 & $\mathbf{4 9 . 5 9 5 1}$ & 37.0445 & 33.1984 \\
NIQE & & & & & & & \\
SSIM & 7.3487 & 5.6763 & 5.4389 & 5.0839 & 7.0417 & 4.7936 & $\mathbf{4 . 1 8 3 2}$ \\
UQI $^{\mathrm{a}}$ & 0.7684 & 0.7876 & 0.8121 & - & 0.9112 & 0.7287 & $\mathbf{0 . 7 1 8 1}$ \\
\hline \hline
\end{tabular}

aHigher value represents a lower quality.

Table 4 The objective image quality comparison of dehazing results of Fig. 27 with different evaluation indexes.

\begin{tabular}{|c|c|c|c|c|c|c|c|}
\hline Quality evaluation & $\mathrm{DCP}$ & Meng & MSRCR & Tarel & DehazeNet & RGB PDM & HSI PDM \\
\hline$e$ & 12.4207 & 38.3588 & 9.5969 & 35.0644 & 7.8212 & 39.041 & 39.3792 \\
\hline $\bar{r}$ & 1.3419 & 2.7161 & 1.9169 & 2.91 & 1.3482 & 2.8812 & 2.9927 \\
\hline IVM & 3.4179 & 11.0702 & 2.909 & 8.2451 & 2.3648 & 4.6748 & 4.7851 \\
\hline Contrast gain & 0.0987 & 0.1878 & 0.1319 & 0.1903 & 0.0611 & 0.1861 & 0.1951 \\
\hline VCM & 26.9512 & 80 & 34.6341 & 36.0976 & 25.3659 & 34.7561 & 50.122 \\
\hline $\mathrm{NIQE}^{\mathrm{a}}$ & 6.9146 & 5.6518 & 5.3835 & 5.3769 & 6.1311 & 3.2395 & 2.7853 \\
\hline $\operatorname{SSIM}^{a}$ & 0.8793 & 0.7788 & 0.8844 & 0.7935 & 0.9504 & 0.7248 & 0.6989 \\
\hline UQI $^{\mathrm{a}}$ & 0.8366 & 0.7205 & 0.9406 & 0.9159 & 0.9433 & 0.7343 & 0.7166 \\
\hline
\end{tabular}

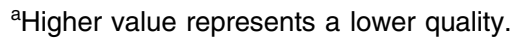

compare above dehazing methods, and the comparison results are shown in Tables $2-4$ corresponding to Figs. 25-27. The comparison results show that the polarimetric dehazing methods outperform the single image dehazing methods in most of the evaluation indexes, although these evaluation indexes assess the image through different characteristics. This may be the intrinsic advantage, because more input images contain much more information about the scenes.

\section{Conclusions}

In this work, we present the image degradation model caused due to haze. This model is widely used in the computer vision and dehazing applications. The polarimetric dehazing methods are based on this degradation model. These methods make an effort to restore the object's light and enhance the image visibility by restoring the information. The basic principle of the polarimetric dehazing method is to estimate the airlight radiance with multiple polarization images, which shows that the partially linearly polarized property is determined by Mie scattering theory. We present a complete review of the techniques, the advancements, the implementation methods, and the algorithms of the polarimetric dehazing methods. Experimental results further verified 
the dehazing capacity of the polarimetric dehazing methods. We believe that this review can significantly assist the overall understanding of the polarimetric dehazing methods.

\section{Acknowledgments}

The authors thank Shandong Provincial Natural Science Foundation, China (ZR2020QA066, ZR2020MA087) and the National Natural Science Foundation of China (NSFC) (11904213, 11704226).

\section{References}

1. R. C. Henry et al., "Colour perception through atmospheric haze," J. Opt. Soc. Am. A 17(5), 831-835 (2000).

2. S. G. Narasimhan and S. K. Nayar, "Vision and the atmosphere," Int. J. Comput. Vision 48(3), 233-254 (2002).

3. T. K. Kim, J. K. Paik, and B. S. Kang, "Contrast enhancement system using spatially adaptive histogram equalization with temporal filtering," IEEE Trans. Consum. Electron. 44(1), 82-87 (1998).

4. J. Y. Kim, L. S. Kim, and S. H. Hwang, "An advanced contrast enhancement using partially overlapped sub-block histogram equalization," IEEE Trans. Circuits Syst. Video Technol. 11(4), 475-484 (2001).

5. M. Kim and M. Chung, "Recursively separated and weighted histogram equalization for brightness preservation and contrast enhancement," IEEE Trans. Consum. Electron. 54(3), 1389-1397 (2008).

6. L. J. Wang and R. Zhu, "Image defogging algorithm of single color image based on wavelet transform and histogram equalization," Appl. Math. Sci. 7(79), 3913-3921 (2013).

7. D. J. Jobson, Z. Rahman, and G. A. Woodell, "A multiscale retinex for bridging the gap between color images and the human observation of scenes," IEEE Trans. Image Process. 6(7), 965-976 (1996).

8. D. J. Jobson, Z. Rahman, and G. A. Woodell, "Properties and performance of a center/ surround retinex," IEEE Trans. Image Process. 6(3), 451-462 (1997).

9. Z. Rahman, D. J. Jobson, and G. A. Woodell, "Retinex processing for automatic image enhancement," Proc. SPIE 4662, 100-110 (2004).

10. C. Busch and E. Debes, "Wavelet transform for analyzing fog visibility," IEEE Intell. Syst. Appl. 13(6), 66-71 (1998).

11. S. Dippel et al., "Multiscale contrast enhancement for radiographies: Laplacian pyramid versus fast wavelet transform," IEEE Trans. Med. Imaging 21(4), 343-353 (2002).

12. R. Zhu and L. J. Wang, "Improved wavelet transform algorithm for single image dehazing," Optik 125(13), 3064-3066 (2014).

13. M. J. Seow and V. K. Asari, "Ratio rule and homomorphic filter for enhancement of digital colour image," Neurocomputing 69 (7/9), 954-958 (2006).

14. P. Carr and R. Hartley, "Improved single image dehazing using geometry," in Proc. IEEE Conf. Digital Image Comput.: Tech. and Appl., IEEE, pp. 103-110 (2009).

15. C. O. Ancuti, C. Ancuti, and P. Bekaert, "Effective single image dehazing by fusion," in Proc. IEEE Conf. Image Process., IEEE, pp. 3541-3544 (2010).

16. P. U. Naik and S. Borkar, "Image dehazing using PCA fusion technique for enhanced road visibility," Int. J. Comput. Appl. 180, 46 (2018).

17. Y. Wang et al., "Haze removal algorithm based on single-images with chromatic properties," Signal Process. Image Commun. 72, 80-91 (2019).

18. R. Luzon-Gonzalez, J. L. Nieves, and J. Romero, "Recovering of weather degraded images based on RGB response ratio constancy," Appl. Opt. 54(4), B222-B231 (2015).

19. S. Salazar-Colores et al., "A fast image dehazing algorithm using morphological reconstruction," IEEE Trans. Image Process. 28(5), 2357-2366 (2019).

20. R. Fattal, "Single image dehazing," ACM Trans. Graphics 27(3), 1-9 (2008). 
21. J. Tarel and N. Hautiere, "Fast visibility restoration from a single colour or gray level image," in Proc. IEEE Conf. Comput. Vision, IEEE, pp. 2201-2208 (2009).

22. R. T. Tan, "Visibility in bad weather from a single image," in Proc. Conf. Comput. Vision and Pattern Recognit., IEEE, pp. 1-7 (2008).

23. K. M. He, J. Sun, and X. O. Tang, "Single image haze removal using dark channel prior," IEEE Trans. Pattern Anal. Mach. Intell. 33(12), 2341-2353 (2011).

24. K. M. He, J. Sun, and X. O. Tang, "Guided image filtering," IEEE Trans. Pattern Anal. Mach. Intell. 35(6), 1397-1409 (2013).

25. W. Huang, Y. Y. Wang, and R. Wang, "A high fidelity haze removal algorithm for optical satellite images using progressive transmission estimation based on the dark channel prior," Int. J. Remote Sens. 40, 3486-3503 (2018).

26. G. Meng et al., "Efficient image dehazing with boundary constraint and contextual regularization," in Proc. IEEE Conf. Comput. Vision, IEEE, pp. 617-624 (2013).

27. L. Kratz and K. Nishino, "Factorizing scene Albedo and depth from a single foggy image," in Proc. IEEE 12th Conf. Comput. Vision, IEEE, pp. 1701-1708 (2013).

28. K. Nishino, L. Kratz, and S. Lombardi, "Bayesian defogging," Int. J. Comput. Vision 98, 263-278 (2012).

29. H. M. Lu et al., "Single image dehazing through improved atmospheric light estimation," Multimedia Tools Appl. 75, 17081-17096 (2016).

30. C. H. Yeh et al., "Haze effect removal from image via haze density estimation in optical model," Opt. Express 21(22), 27127-27141 (2013).

31. W. Sun and L. Han, "A new fast single image defog algorithm," in Proc. IEEE Conf. Intellect Syst. Des. and Eng. Appl., IEEE, pp. 116-119 (2012).

32. B. Li et al., "AOD-net: all-in-one dehazing network," in the IEEE Int. Conf. Comput. Vision, IEEE, pp. 4780-4788 (2017).

33. B. Cai et al., "DehazeNet: an end-to-end system for single image haze removal," IEEE Trans. Image Process. 25(11), 5187-5198 (2016).

34. Y. Xu et al., "Review of video and image defogging algorithms and related studies on image restoration and enhancement," IEEE Access 4, 165-188 (2016).

35. S. G. Narasimhan and S. K. Nayar, "Removing weather effects from monochrome images," in Proc. IEEE Comput. Soc. Conf. Comput. Vision and Pattern Recognit., IEEE, Vol. 2, pp. II-186-II-193 (2001).

36. S. G. Narasimhan and S. K. Nayar, "Chromatic framework for vision in bad weather," in Proc. IEEE Conf. Comput. Vision and Pattern Recognit., IEEE, Vol. 1, pp. 598-605 (2000).

37. S. G. Narasimhan and S. K. Nayar, "Contrast restoration of weather degraded images," IEEE Trans. Pattern Anal. Mach. Learn. 25(6), 713-724 (2003).

38. S. G. Narasimhan and S. K. Nayar, "Interactive (de)weathering of an image using physical models," in Proc. IEEE Workshop Color Photometric Methods Comput. Vision, IEEE, Vol. 6, pp. 1-8 (2003).

39. L. Schaul, C. Fredembach, and S. Susstrunk, "Color image dehazing using the nearinfrared," in Proc. IEEE Conf. Image Process., IEEE, pp. 1-4 (2009).

40. C. Feng et al., "Near-infrared guided color image dehazing," in Proc. IEEE Conf. Image Process., IEEE, pp. 2363-2367 (2013).

41. D. B. Chenault and J. L. Pezzaniti, "Polarization imaging through scattering media," Proc. SPIE 4133, 124-133 (2000).

42. Y. Y. Schechner, S. G. Narasimhan, and S. K. Nayar, "Instant dehazing of images using polarization," in Proc. IEEE Conf. Comput. Vision and Pattern Recognit., IEEE, pp. 325$332(2001)$.

43. Y. Y. Schechner, S. G. Narasimhan, and S. K. Nayar, "Polarization-based vision through haze," Appl. Opt. 42(3), 511-525 (2003).

44. Y. Y. Schechner and N. Karpel, "Recovering scenes by polarization analysis," in IEEE Techno-Ocean 04CH37600 (2004).

45. T. Treibitz and Y. Y. Schechner, "Polarization: beneficial for visibility enhancement?" in Proc. IEEE Conf. Comput. Vision and Pattern Recognit., IEEE, pp. 525-532 (2009). 
46. E. Namer and Y. Y. Schechner, "Advanced visibility improvement based on polarization filtered images," Proc. SPIE 5888, 588805 (2005).

47. E. Namer, S. Shwartz, and Y. Y. Schechner, "Skyless polarimetric calibration and visibility enhancement," Opt. Express 17(2), 472-493 (2009).

48. J. Fade et al., "Long-range polarimetric imaging through fog," Appl. Opt. 53(18), 38543865 (2014).

49. J. Mudge and M. Virgen, "Real time polarimetric dehazing," Appl. Opt. 52(9), 1932-1938 (2013).

50. J. Liang et al., "Method for enhancing visibility of hazy images based on polarimetric imaging," Photonics Res. 2(1), 38-44 (2014).

51. J. Liang et al., "Visibility enhancement of hazy images based on a universal polarimetric imaging method," J. Appl. Phys. 116(17), 173107 (2014).

52. X. Li et al., "Imaging through haze utilizing a multi-aperture coaxial polarization imager," in Front. Opt., Optical Society of America, JW4A-136 (2018).

53. M. E. Ketara and S. Breugnot, "Imaging through haze using multispectral polarization imaging method," Proc. SPIE 10655, 106550N (2018).

54. M. J. Raković et al., "Light backscattering polarization patterns from turbid media: theory and experiment," Appl. Opt. 38(15), 3399-3408 (1999).

55. Y. Y. Schechner and N. Karpel, "Recovery of underwater visibility and structure by polarization analysis," IEEE J. Oceanic Eng. 30(3), 570-587 (2005).

56. J. Shen et al., "Polarization calculation and underwater target detection inspired by biological visual imaging," Sens. Transd. 169(4), 33-41 (2014).

57. S. Fang et al., "Image dehazing using polarization effects of objects and airlight," Opt. Express 22(16), 19523-19538 (2014).

58. W. Zhang et al., "A robust haze-removal scheme in polarimetric dehazing imaging based on automatic identification of sky region," Opt. Laser Technol. 86, 145-151 (2016).

59. W. F. Zhang et al., "Study of visibility enhancement of hazy images based on dark channel prior in polarimetric imaging," Optik 130, 123-130 (2017).

60. W. F. Zhang et al., "Real-time image haze removal using an aperture-division polarimetric camera," Appl. Opt. 56(4), 942-947 (2017).

61. Y. Tian et al., "Underwater imaging based on LF and polarization," IEEE Photonics J. 11, 6500309 (2019).

62. W. F. Zhang, J. Liang, and L. Y. Ren, "Haze-removal polarimetric imaging schemes with the consideration of airlight's circular polarization effect," Optik 182, 1099-1105 (2019).

63. L. Zhang et al., "Lane detection in dense fog using a polarimetric dehazing method," Appl. Opt. 59(19), 5702-5707 (2020).

64. J. Liang et al., "Generalized polarimetric dehazing method based on low-pass filtering in frequency domain," Sensors 20(6), 1729 (2020).

65. Z. Liang et al., "Effective polarization-based image dehazing with regularization constraint," IEEE Geosci. Remote Sens. Lett., 1-5 (2020).

66. X. P. Fu et al., "Image descattering and absorption compsnsation in underwater polarimetric imaging," Opt. Lasers Eng. 132, 106115 (2020).

67. H. F. Hu et al., "Polarimetric underwater image recovery via deep learning," Opt. Lasers Eng. 133, 106152 (2020).

68. F. Liu et al., "Polarimetric dehazing utilizing spatial frequency segregation of images," Appl. Opt. 54(27), 8116-8122 (2015).

69. S. Shwartz, E. Namer, and Y. Y. Schechner, "Blind haze separation," in Proc. Conf. Comput. Vision and Pattern Recognit., IEEE, pp. 1984-1991 (2006).

70. R. Kaftory, Y. Y. Schechner, and Y. Y. Zeevi, "Variational distance-dependent image restoration," in Proc. IEEE Conf. Comput. Vision and Pattern Recognit., IEEE, pp. 1-8 (2007).

71. B. J. Huang et al., "Underwater image recovery considering polarization effects of objects," Opt. Express 24(9), 9826-9838 (2016).

72. J. G. Guan et al., "Real-time polarization difference underwater imaging based on Stokes vector," Acta Phys. Sin. 64, 224203 (2015).

73. P. C. Y Chang et al., "Improving visibility depth in passive underwater imaging by use of polarization," Appl. Opt. 42(15), 2794-2803 (2003). 
74. F. Wang, C. H. Yin, and Y. Wang, "Research of polarization imaging detection method for water surface target in foggy weather," Proc. SPIE 8907, 89074C (2013).

75. J. G. Guan and J. P. Zhu, "Target detection in turbid medium using polarization-based range-gated technology," Opt. Express 21(12), 14152-14158 (2013).

76. Y. Y. Schechner and N. Karpel, "Clear underwater vision," in Proc. IEEE Conf. Comput. Vision and Pattern Recognit., IEEE, pp. 536-543 (2004).

77. A. Sarafraz, S. Negahdaripour, and Y. Y. Schechner, "Enhancing images in scattering media utilizing stereovision and polarization," in IEEE Workshop Appl. Comput. Vision, IEEE, pp. 1-8 (2009).

78. D. Brousseau, J. Plant, and S. Thibault, "Real-time polarization difference imaging (PDI) reveals surface details and textures in harsh environments," Proc. SPIE 8720, 87200E (2013).

79. W. J. Zhang et al., "Sky light polarization detection with linear polarizer triplet in light field camera inspired by insect vision," Appl. Opt. 54(30), 8962-8970 (2015).

80. D. H. Goldstein, Polarized Light, 3rd ed., Taylor and Francis Group, New York (2011).

81. J. Liang et al., "Polarimetric dehazing method for dense haze removal based on distribution analysis of angle of polarization," Opt. Express 23(20), 26146-26157 (2015).

82. C. X. Zhao et al., "Experimental comparison of polarization image restoration of three random angles and two orthogonal angles," Laser Optoelectron. Prog. 52, 101005 (2015).

83. L. Cao et al., "Dehazing method through polarimetric imaging and multi-scale analysis," Proc. SPIE 9501, 950111 (2015).

84. Y. Q. Zhao, Q. Pan, and H. C. Zhang, "New polarization imaging method based on spatially adaptive wavelet image fusion," Opt. Eng. 45(12), 123202 (2006).

85. Y. Wang, M. G. Xue, and Q. C. Huang, "Polarization dehazing algorithm based on atmosphere background suppression," Comput. Eng. 35(4), 271-275 (2009).

86. W. Z. Peng, "Polarization dehazing algorithm based on atmosphere scattering model," Electron. Meas. Technol. 34(7), 43-45 (2011).

87. X. L. Zhang, Y. Xu, and X. Z. Wang, "Research on image fusion based on polarization of haze," J. Xiamen Univ. 50(3), 520-524 (2011).

88. J. Liang et al., "Polarimetric dehazing method for visibility improvement based on visible and infrared image fusion," Appl. Opt. 55(29), 8221-8226 (2016).

89. W. F. Zhang et al., "Fast polarimetric dehazing method for visibility enhancement in HSI colour space," J. Opt. 19, 095606 (2017).

90. M. Ebner, Colour Constancy, John Wiley \& Sons Ltd., Hoboken (2007).

91. J. S. Tyo et al., "Review of passive imaging polarimetry for remote sensing applications," Appl. Opt. 45(22), 5453-5469 (2006).

92. N. Lefaudeux et al., "Compact and robust linear Stokes polarization camera," Proc. SPIE 6972, 69720B (2008).

93. A. Jaulin and L. Bigue, "High speed partial Stokes imaging using a ferroelectric liquid crystal modulator," J. Eur. Opt. Soc.-Rapid Publ. 3, 08019 (2008).

94. P. Mukherjee et al., "Implementation of a complete Muller matrix polarimeter using dual photoelastic modulators and rotating wave plates," Opt. Rev. 26, 23-32 (2019).

95. M. Vedel, S. Breugnot, and N. Lechocinski, "Full Stokes polarization imaging camera," Proc. SPIE 8160, 81600X (2011).

96. J. Mudge, M. Virgen, and P. Dean, "Near-infrared simultaneous Stokes imaging polarimeter," Proc. SPIE 7461, 74610L (2009).

97. X. Li et al., "Research on polarization dehazing through the coaxial and multi-aperture polarimetric camera," OSA Continuum 2(8), 2369-2380 (2019).

98. R. Perkins and V. Gruev, "Signal-to-noise analysis of Stokes parameters in division of focal plane polarimeters," Opt. Express 18(25), 25815-25824 (2010).

99. G. Myhre et al., "Liquid crystal polymer full-Stokes division of focal plane polarimeter," Opt. Express 20(25), 27393-27409 (2012).

100. C. H. Xu et al., "Numerical study of a DoFP polarimeter based on the self-organized nanograting array," Opt. Express 26(3), 2517-2527 (2018).

101. K. Oka and N. Saito, "Snapshot complete imaging polarimeter using Savart plates," Proc. SPIE 6295, 629508 (2006). 
102. H. T. Luo et al., "Compact and miniature snapshot imaging polarimeter," Appl. Opt. 47(24), 4413-4417 (2008).

103. M. Honma, N. Takahashi, and T. Nose, "Simple Stokes polarimeter using liquid crystal grating with ternary orientation domains," Appl. Opt. 57(35), 10183-10190 (2018).

104. J. D. Perreault, "Triple Wollaston-prism complete-Stokes imaging polarimeter," Opt. Lett. 38(19), 3874-3877 (2013).

105. M. M. Ali, M. A. U. Rahman, and S. Hajera, "A comparative study of various image dehazing techniques," in Int. Conf. Energy, Communication, Data Analytics and Soft Comput., pp. 3622-3628 (2017).

106. N. Hautière et al., "Blind contrast enhancement assessment by gradient ratioing at visible edges," Image Anal. Stereol. J. 27(2), 87-95 (2008).

107. X. Yu et al., "A classification algorithm to distinguish image as haze or non-haze," in Proc. IEEE Int. Conf. Image Graphics, IEEE, pp. 286-289 (2011).

108. T. L. Economopoulos, P. A. Asvestas, and G. K. Matsopoulos, "Contrast enhancement of images using partitioned iterated function systems," Image Vision Comput. 28(1), 45-54 (2010).

109. D. J. Jobson et al., "A comparison of visual statistics for the image enhancement of FORESITE aerial images with those of major image classes," Proc. SPIE 6246, 624601 (2006).

110. A. Mittal, R. Soundararajan, and A. C. Bovik, "Making a "completely blind" image quality analyzer," IEEE Signal Process. Lett. 20(3), 209-212 (2013).

111. Z. Wang and A. C. Bovik, "A universal image quality index," IEEE Signal Process. Lett. 9 (3), 81-84 (2002).

Wenfei Zhang received his BS degree in applied physics in 2009 and his MS degree in condensed physics in 2012 from Qingdao University, and his PhD in electric science and technology from Xi'an Jiaotong University, China. His current work is the polarimetric imaging and polarimetric dehazing methods in turbid media.

Jian Liang received his $\mathrm{PhD}$ in optics from Xi' an Institute of Optics and Precision Mechanics, Chinese Academy of Sciences, China. His current work is polarimetric imaging, polarimetric dehazing methods in turbid media, and the polarimetric imaging systems.

Guomei Wang received her $\mathrm{PhD}$ in optics from Xi'an Institute of Optics and Precision Mechanics, Chinese Academy of Sciences, China. Her current work is the fiber laser technique.

Huanian Zhang received his $\mathrm{PhD}$ from Shandong University, China. His current research interest is the ultrafast fiber laser and its application

Shenggui Fu received his MS and PhD degrees from Nankai University, China. He is a postdoctor at Nankai University. His current research interest is the laser technology and optical fiber photonics. 